Draft Version August 2, 2021

Preprint typeset using $\mathrm{IATE}_{\mathrm{E}} \mathrm{X}$ style emulateapj v. 08/13/06

\title{
A TALE OF TWO ANOMALIES: DEPLETION, DISPERSION, AND THE CONNECTION BETWEEN THE STELLAR LITHIUM SPREAD AND INFLATED RADII ON THE PRE-MAIN SEQUENCE
}

\author{
Garrett Somers and Marc H. Pinsonneault \\ Draft version August 2, 2021
}

\begin{abstract}
We investigate lithium depletion in standard stellar models (SSMs) and main sequence (MS) open clusters, and explore the origin of the $\mathrm{Li}$ dispersion in young, cool stars of equal mass, age and composition. We first demonstrate that SSMs accurately predict the Li abundances of solar analogs at the zero-age main sequence (ZAMS) within theoretical uncertainties. We then measure the rate of MS Li depletion by removing the $[\mathrm{Fe} / \mathrm{H}]$-dependent ZAMS Li pattern from three well-studied clusters, and comparing the detrended data. MS depletion is found to be mass dependent, in the sense of more depletion at low mass. A dispersion in Li abundance at fixed $T_{\text {eff }}$ is nearly universal, and sets in by $\sim 200$ Myr. We discuss mass and age dispersion trends, and the pattern is mixed. We argue that metallicity impacts the ZAMS Li pattern, in agreement with theoretical expectations but contrary to the findings of some previous studies, and suggest Li as a test of cluster metallicity. Finally, we argue that a radius dispersion in stars of fixed mass and age, during the epoch of pre-MS Li destruction, is responsible for the spread in $\mathrm{Li}$ abundances and the correlation between rotation and $\mathrm{Li}$ in young cool stars, most well known in the Pleiades. We calculate stellar models, inflated to match observed radius anomalies in magnetically active systems, and the resulting range of Li abundances reproduces the observed patterns of young clusters. We discuss ramifications for pre-MS evolutionary tracks and age measurements of young clusters, and suggest an observational test.
\end{abstract}

Subject headings:

\section{INTRODUCTION}

The lithium content of stars is an important quantity for a variety of astrophysical measurements. First, Li is a powerful tracer of mixing in stars. It is destroyed efficiently at $\sim 2.5$ million $\mathrm{K}\left(T_{L B}\right)$, and as a result can only survive in the outer layers of stars. When a star is undergoing deep mixing, Li-depleted stellar material is transported from depths that surpass $T_{L B}$ to the surface, diluting the observed $\mathrm{Li}$ abundance $(\mathrm{A}(\mathrm{Li})=12+[\mathrm{Li} / \mathrm{H}])$. The evolution of $\mathrm{Li}$ in stellar atmospheres is therefore a direct consequence of mixing, which in turn affects the surface composition and main-sequence (MS) lifetimes of stars across the stellar mass function (e.g. Pinsonneault 1997). Second, the evolution of $\mathrm{Li}$ abundances on the pre-MS and MS contains information about stellar ages (e.g. Jeffries 2000), and may inform our knowledge about their rotational history (Pinsonneault 1990). Finally, the $\mathrm{Li}$ content of the universe is a strong prediction of big bang nucleosynthesis (Boesgaard \& Steigman 1985), and can be probed by measuring the initial $\mathrm{Li}$ abundance of very old stars in the Galaxy (e.g. Spite \& Spite 1982; Cyburt et al. 2008).

Interiors models make strong predictions about the timescales of mixing, and thus the evolution of surface $\mathrm{Li}$, as a function of mass, age, and composition. Because the sole mixing mechanism in standard stellar models (SSMs) is convection, the surface $\mathrm{Li}$ abundance of a star is predicted to decrease only when the temperature at the base of the surface convection zone $\left(T_{B C Z}\right)$ is greater than $T_{L B}$. This occurs on the pre-MS for stars of mass $0.5 M_{\odot}-1.3 M_{\odot}$ at solar metallicity, but not on the MS (Iben 1965). The general theoretical expectations of preMS Li depletion in this mass range are well established, and a qualitative explanation follows. Pre-MS stars have deep convective envelopes, and heat up as they contract, causing $T_{B C Z}$ to eventually surpasses $T_{L B}$ and inducing Li depletion. In fully convective stars (FCSs; $M_{\odot}<$ $\left.0.35 M_{\odot}\right), \mathrm{Li}$ is completely destroyed in only a few $\mathrm{Myr}$ once $T_{L B}$ is reached. This occurs earlier for higher mass objects, creating a boundary between FCSs which have depleted Li, and lower mass FCSs which remain Li-rich. The location of this boundary is age dependent, permitting its use as a method for dating clusters; this is called the lithium depletion boundary (LDB) technique (Basri et al. 1996; Bildsten et al. 1997). Stars less massive than $\sim 0.06 M_{\odot}$ never reach $T_{L B}$ in their interior, and so retain their initial $\mathrm{Li}$ abundance forever.

For stars more massive than $0.35 M_{\odot}$, the convective envelope begins to retreat on the late pre-MS; this causes $T_{B C Z}$ to once again cool below $T_{L B}$, and terminates $\mathrm{Li}$ depletion. Lower mass stars take longer to reach $T_{L B}$, but remain in the burning phase for longer. This results in greater depletion factors in these objects. Li also burns more rapidly in metal-rich stars, as the higher resulting opacity deepens the convective envelope, increasing $T_{B C Z}$. The result is a strongly mass and metallicity dependent lithium depletion pattern (LDP) on the zeroage MS (ZAMS) with no dispersion at fixed mass, in accordance with the Vogt-Russell theorem.

For a solar metallicity cluster, Li has been completely destroyed in stars $\lesssim 0.6 M_{\odot}$ by the zero-age MS (ZAMS), but only about $\sim 20 \%$ has been destroyed for $1.3 M_{\odot}$. Once on the MS, the $\mathrm{Li}$ depletion zone resides inside the radiative core, and SSMs predict no more mixingrelated Li depletion until the MS turn-off. The LDP will continue to change on Gyr timescales, but this is not a result of mixing, and is instead due to the evolving temperature of MS stars, and gravitational settling in late $\mathrm{F}$ and early $\mathrm{G}$ dwarfs. 


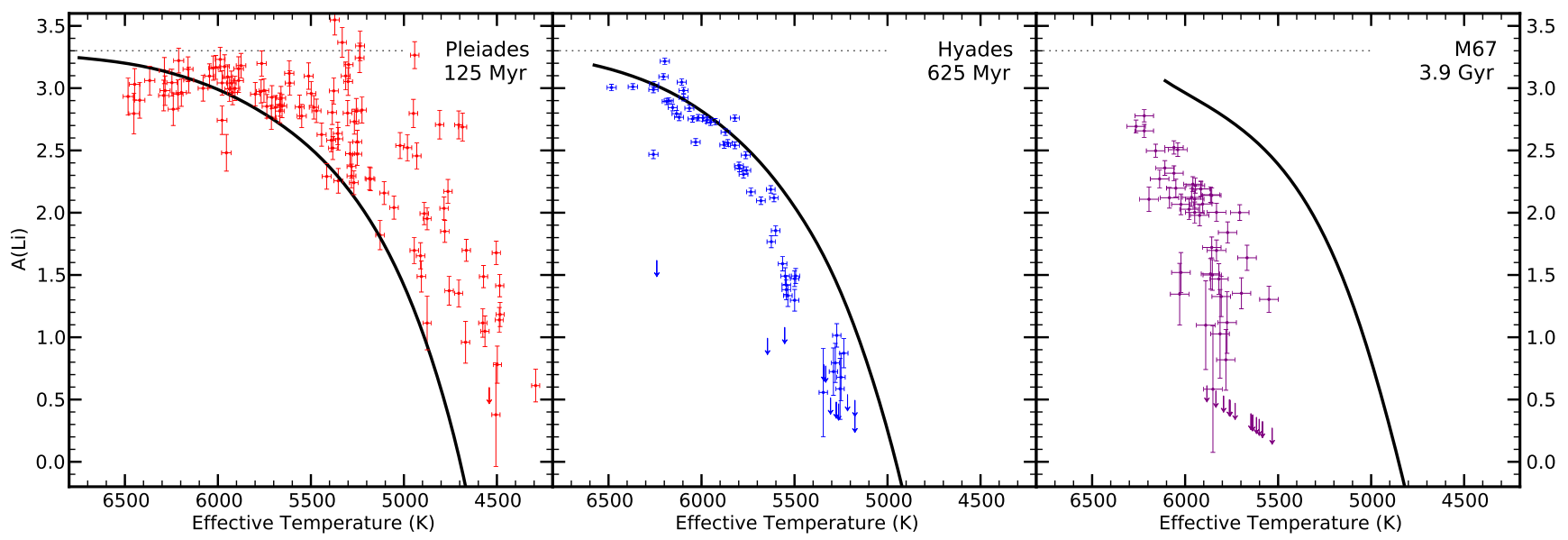

FIG. 1.- Lithium data for the Pleiades, Hyades, and M67 are shown alongside standard stellar model lithium patterns calculated for their respective cluster parameters. The solid lines represent the SSM prediction for each cluster, and the dashed line in the top right panel represents the M67 LDP at the age of the Pleiades, demonstrating the MS evolution of LDPs in the standard model. This is almost entirely due to the increasing $T_{\text {eff }}$ on the MS, but also includes minor gravitational settling. Arrow denote upper limits.

Many Li data sets exist in the literature, but the important features of the general observational picture can be accurately represented by three well-studied clusters. The top panel of Fig. 1 shows empirical Li data for the Pleiades, Hyades, and M67, alongside SSM LDPs calculated for their respective cluster parameters (solid black lines; see $§ 2.1-2.3$ and Table 1 for details). As illustrated by this figure, there are several inconsistencies between standard predictions and observed cluster patterns:

(i) The median abundance is over-predicted by a few tenths of a dex above $6100 \mathrm{~K}$, and under-predicted by a few tenths of dex to greater than an order-of-magnitude below $6100 \mathrm{~K}$. While the general trend of greater depletion in cooler stars is accurately predicted, this SSM marginally fails to predict the median of solar analogs, and catastrophically fails to predict the median of cool stars.

(ii) There is a significant scatter in surface Li in the cool $\left(T_{\text {eff }}<5500 \mathrm{~K}\right)$ Pleiads, where the width of this distribution can be as large as a factor of 100 . This implies that additional physical parameters, which can vary between equal- $T_{\text {eff }}$ stars, affects pre-MS depletion by orders-of-magnitude in this temperature regime. Furthermore, the fastest rotating cool Pleiads are on average the most Li-rich stars at their respective temperatures (Soderblom et al. 1993a; S93 hereafter). This strongly implies a connection between rotation and $\mathrm{Li}$ depletion.

(iii) Fig. 1 shows a strong temporal evolution of the median Li abundance at all masses, occurring on a much shorter timescale than MS evolution. From left to right, SSMs first under-predict, then over-predict, then greatly over-predict the median pattern at $100 \mathrm{Myr}, 600 \mathrm{Myr}$, and 4 Gyr. This implies that mechanisms other than convection are able to mix stellar material on the MS. Furthermore, the rate of depletion decreases at advanced ages (e.g. Sestito \& Randich 2005; SR05 hereafter).

(iv) By the age of M67, a large Li scatter has developed in solar analogs. Such a scatter is not present in the Pleiades, and so likely develops during the MS. This demonstrates that the Li abundance of a given star depends on factors other than just mass, age, and metallicity. Additional physical parameters that vary between equal-mass stars must induce this relative depletion.
These inconsistencies demonstrate that, in contrast to standard theory, MS LDPs are the product of two distinct processes: a pre-MS process that imparts a strongly mass-dependent median trend with a variable width, and a longer timescale processes on the MS that causes the median and dispersion to evolve with time. In this paper we will deal, in some part, with both of these processes. For the former, we will validate the accuracy of SSMs in warm stars, argue the importance of metallicity in shaping ZAMS Li patterns, and propose an explanation for the dispersion in cool stars. For the latter, we will produce an empirical measure of MS Li depletion that has been corrected for metallicity effects, and study the timescales of the emergence of $\mathrm{Li}$ dispersion on the MS. These can be used both to anchor mixing calculations on empirical data and to place constraints on proposed mixing mechanisms. In all cases, we will refer to the difference between SSM predictions, and the empirical abundance of stars, as the lithium anomaly.

Li depletion on the MS has be known about for several decades (e.g. Herbig 1965; Zappala et al. 1972; Balachandran 1995; Pinsonneault 1997; SR05), but the mechanism, or mechanisms, responsible have yet to be definitively established. Suspects include mixing driven by rotation and angular momentum (AM) loss (Pinsonneault et al. 1989; Zahn 1992; Chaboyer et al. 1995), mixing driven by internal gravity waves (Press 1981; Montalban \& Schatzman 2000), dilution of the envelope through mass loss (Swenson \& Faulkner 1992), and microscopic diffusion (Richer \& Michaud 1993). Rotational mixing is a particularly promising explanation for two reasons. First, stellar rotation slows over time (Skumanich 1972), naturally explaining the decay of Li depletion rates described in (iii). Second, stellar rotation rates show a large dispersion at ZAMS (e.g. Stauffer et al. 1984), providing the necessary variant between stars of equal mass described in $(i v)$. We will present updated models of rotationally-induced mixing in a forthcoming paper (Somers \& Pinsonneault 2014b, in prep; Paper II hereafter). However, before we can perform precision tests, accurate measurements of this depletion must be obtained.

While previous authors have measured MS Li depletion 
TABLE 1

Benchmark Cluster Data

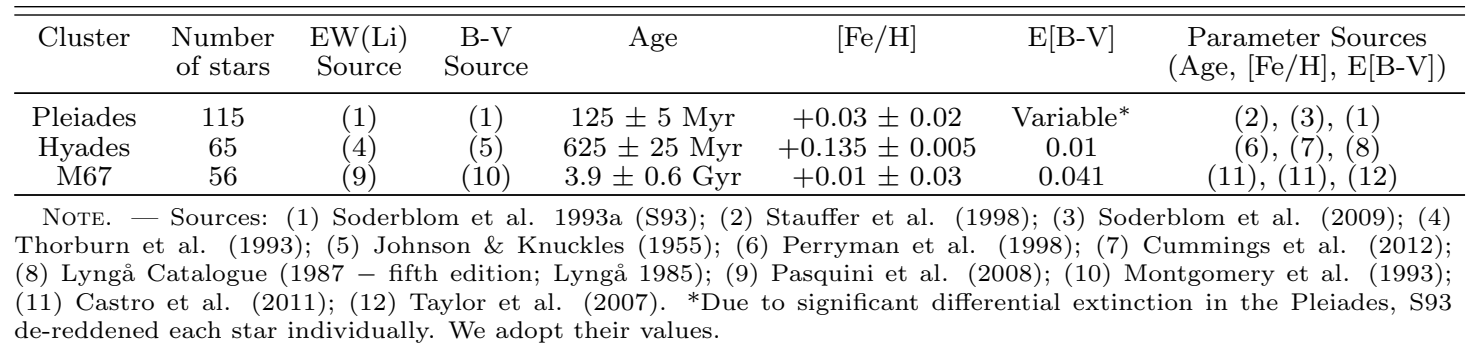

by comparing the abundances of different-aged MS clusters (e.g. SR05), these studies have not accounted for one crucial element: higher metallicity stars are expected to deplete greater amounts of Li during the pre-MS. This effect can severely bias comparisons in absolute space, as the ZAMS abundance at a given $T_{\text {eff }}$ may differ between clusters by up to an order of magnitude (§3.1). To address this, we present a novel method in $\S 4$ for quantifying the lithium anomaly that develops on the MS. We will argue that the MS anomaly signal can be isolated from an empirical MS Li pattern by subtracting a SSM LDP from the data. This removes the relative, $[\mathrm{Fe} / \mathrm{H}]$-dependent pre-MS depletion signal, leaving behind the depletion induced by non-standard MS mixing. Although some authors have claimed that this metallicity effect is not supported by observational evidence, we present a case in $\S 6.1$ that composition is indeed central in shaping ZAMS Li patterns.

The efficacy of this method hinges on the quality of SSM LDP predictions, which we know from (i) can be poor. Therefore, we must first reconcile our theoretical predictions with the data. To do this, we explore the possibility that errors in model input physics account for this discrepancy. The extreme sensitivity of the rate of Li burning to the surrounding temperature $\left(\propto T^{20}\right.$; Bildsten et al. 1997) implies that minute changes in $T_{B C Z}$ on the pre-MS may have large effects on the magnitude of Li depletion predicted in SSMs. $T_{B C Z}$ in a stellar model may be affected by the assumed physics, so in order to validate this method, we first address the following question: can SSMs accurately predict the magnitude of pre-MS Li depletion within the errors of our adopted input physics? If this is so, we can use empirical data to calibrate our SSMs, and produce accurate predictions of pre-MS Li depletion ( $(4)$.

We also investigate one of the key outstanding problems in our understanding of pre-MS depletion: the cool star Li dispersion in the Pleiades and other young systems (S93). It is unlikely that long timescale mechanisms such as rotationally induced mixing are responsible for this dispersion, given its early onset. However, the rotation-Li correlation in young Pleiads, described in (ii), suggests either a causal or corollary relationship between rotation and the efficiency of early convective depletion. Another effect known to correlate with rotation in stars is the so-called radius anomaly. This describes a discrepancy of $\sim 5-15 \%$ the observed radii of some stars, and their SSM predictions. The radius anomaly has been observed in detached eclipsing binaries (DEBs; Popper 1997; Torres \& Ribas 2002; Ribas 2003; López-Morales \& Ribas 2005, López-Morales 2007; Torres et al. 2010;
Kraus et al. 2011; Irwin et al. 2011; Feiden \& Chaboyer 2012, Stassun et al. 2012), and may be present in interferometric radius measurements of single field stars (Berger et al. 2006; Boyajian et al. 2008; Boyajian et al. 2012; but, see Demory et al. 2009). This effect has also been reported in solar analogs (e.g. Clausen et al. 2009), and in pre-MS stars (Stassun et al. 2006; Stassun et al. 2007). The latter authors discovered a brown dwarf binary system where the more massive object has a lower $T_{\text {eff }}$. Temperature anomalies correlate with radius anomalies, definitively showing that coeval objects can be differentially affected by non-standard stellar parameters. This radius effect may be caused by accretion from a circumstellar disk (Palla \& Stahler 1992), unidentified sources of opacity (Berger et al. 2006), or inhibition of convection by magnetic activity (Mullan \& MacDonald 2001; Chabrier et al. 2007; Morales et al. 2008; MacDonald \& Mullan 2012; Feiden \& Chaboyer 2013). Although the two fields of radius anomalies and open cluster $\mathrm{Li}$ data have not previously intersected, we reveal a surprising connection between them in $\S 6.2$.

The rest of the text is organized as follows. In $\S 2.1$, we describe the open cluster Li data sets used for our analysis. $\$ 2.2$ describes the equivalent width and photometric data we use to infer stellar parameters of our benchmark clusters, and the abundance analysis we employ. $\S 2.3$ describes the stellar evolution code we used to generate theoretical LDPs, and enumerates the physics in our fiducial calculations. We then begin our exploration of uncertainties inherent to the detrending process. In $\S 3.1$, we quantify the impact of $[\mathrm{Fe} / \mathrm{H}]$ errors in our benchmark clusters, and conclude that extremely well-constrained composition is necessary to accurately predict the pre-MS signal. In $§ 3.2$, we perform a systematic study of the effects of various physical inputs on SSM LDPs. We then constrain the input physics in our models with the empirical Pleiades Li pattern, and describe our method of cluster detrending in $\S 4$. In $\S 5.1$, we measure the lithium anomaly in our benchmark clusters, and compare our results to previous calculations. This measurement will ultimately anchor the mixing included in our rotating stellar models (Paper II). We then revisit the data collected in SR05 with our detrending methodology in $\S 5.2$, and explore the evolution of both median $\mathrm{Li}$ abundances and Li dispersion along the MS. In $\S 6.1$, we argue that composition is an important factor in shaping ZAMS LDPs, validate our anomaly measurements, and suggest $\mathrm{Li}$ as a precision test of the metallicity of clusters. Finally, we test an explanation in $\S 6.2$ for the cool star spread in the Pleiades, related to the radii of young, low-mass stars. We conclude by summarizing our 
TABLE 2

Additional Cluster Data

\begin{tabular}{ccccc}
\hline \hline Cluster & $\begin{array}{c}\mathrm{A}(\mathrm{Li}) / T_{\text {eff }} \\
\text { Source }\end{array}$ & $\begin{array}{c}\text { Age } \\
(\mathrm{Myr})\end{array}$ & {$[\mathrm{Fe} / \mathrm{H}]$} & $\begin{array}{c}\text { Parameter Sources } \\
(\text { Age },[\mathrm{Fe} / \mathrm{H}])\end{array}$ \\
\hline NGC 2264 & $(1)$ & $6 \pm 3$ & -0.15 & $(2),(3)$ \\
$\beta$ Pictoris & $(4)$ & $21 \pm 4$ & $0.01 \pm 0.08$ & $(5),(6)$ \\
IC 2602 & $(7)$ & $46 \pm 6$ & $0.00 \pm 0.01$ & $(8),(9)$ \\
NGC 2451 A+B & $(10)$ & $65 \pm 15$ & $-0.01 \pm 0.08$ & $(11),(11)$ \\
$\alpha$ Persei & $(12)$ & $90 \pm 10$ & $-0.05 \pm 0.05$ & $(13),(14)$ \\
Blanco 1 & $(15)$ & $132 \pm 24$ & $0.04 \pm 0.02$ & $(16),(17)$ \\
& & & & \\
\hline
\end{tabular}

Note. - Sources: (1) Soderblom et al. (1999); (2) King et al. (2000); (3) Dahm (2008); (4) Torres et al. (2006); (5) Binks \& Jeffries (2013); (6) Viana Almeida et al. (2009); (7) Randich et al. (2001); (8) Dobbie et al. (2010); (9) D'Orazi et al (2009); (10) Hünsch et al. (2004); (11) Hünsch et al. (2003); (12) Balachandran et al. (2011); (13) Stauffer et al. (1999); (14) Boesgaard \& Friel (1990); (15) Jeffries \& James (1999); (16) Cargile et al. (2010); (17) Ford et al. (2005)

findings and suggesting directions for future studies in $\S 7$.

\section{METHODS}

\subsection{Cluster Selection and Parameters}

Cluster Li data will serve several purposes in this paper. First, we will use three benchmark clusters to precisely measure the rate of MS Li depletion (§2.1.1). The Pleiades will be used to calibrated the physics in our theoretical models, by requiring that its SSM Li pattern agree with its empirical Li pattern at the ZAMS, and the relative abundances of the Hyades and M67 will be used to infer the MS lithium anomaly, by comparing their detrended patterns to those expected from the Pleiades calibration, corrected for their metallicities. Second, we will reanalyze the data of SR05, and examine the timescales of Li depletion and dispersion ( $(5.2)$. Third, we will argue that metallicity plays an important role in pre-MS Li destruction by comparing similar-aged clusters of dissimilar composition ( $(6.1)$. Finally, we will test our pre-MS Li depletion models by comparing their predictions to several additional young clusters $(\S 6.2)$.

\subsubsection{Benchmark Clusters}

The literature hosts a wealth of Li data for FGK dwarfs in open clusters (see SR05 and refs. therein; Torres et al. 2006; Sacco et al. 2007; Prisinzano \& Randich 2007; Pasquini et al. 2008; Randich et al. 2009; Jeffries et al. 2009; Anthony-Twarog et al. 2009; Cargile et al. 2010; Balachandran et al. 2011; Cummings et al. 2012; Pace et al. 2012; François et al. 2013). Li depletion calculations are exquisitely sensitive to composition, so we restrict our potential choices to clusters with small $[\mathrm{Fe} / \mathrm{H}]$ errors $(<0.05$ dex $)$ to minimize uncertainties. This excludes all but the most well-studied clusters. Furthermore, large Li data sets are required to minimize errors resulting from shot noise, because dispersion is a ubiquitous feature.

With these considerations in mind, we select the Pleiades, Hyades, and M67 as our benchmark clusters. These are well-suited for this investigation because they are exceptionally well studied, thus minimizing errors associated with photometry, extinction, binarity, membership, and most importantly, composition. The Pleiades is $125 \pm 5$ Myr old (Stauffer et al. 1998; see Table 1), making it our near ZAMS cluster, The Hyades is $625 \pm$ 25 Myr old (Perryman et al. 1998), and M67 is $3.9 \pm$ 0.6 Gyr old (Castro et al. 2011). This level of temporal coverage allows us to characterize the relative strengths of early and late-time mixing.

\subsubsection{Additional Clusters}

In $\S 5.2$, we will revisit the clusters examined by SR05 through the use of our detrending analysis (see their Table 1 for details). For both the benchmark clusters and those considered in $\S 6.1$, we will adopt the data sets described in this text. For the rest, we adopt the photometry and Li equivalent widths (EWs) reported by SR05, and use the analysis techniques described in $\S 2.2$. We adopt the cluster reddening, ages, and $\mathrm{Fe}$ abundances reported by SR05, except for the following cases, where we have substituted higher resolution metal abundances: $[\mathrm{Fe} / \mathrm{H}]=-0.03 \pm 0.04$ for IC 4665 (Shen et al. 2005), $[\mathrm{Fe} / \mathrm{H}]=0.00 \pm 0.01$ for IC 2602 (D'Orazi et al. 2009), $[\mathrm{Fe} / \mathrm{H}]=-0.01 \pm 0.02$ for IC 2391 (D'Orazi et al. 2009), $[\mathrm{Fe} / \mathrm{H}]=0.04 \pm 0.02$ for Blanco 1 (Ford et al. 2005), $[\mathrm{Fe} / \mathrm{H}]=0.01 \pm 0.07$ for NGC 2516 (Terndrup et al. 2002 ) and $[\mathrm{Fe} / \mathrm{H}]=0.03 \pm 0.02$ for NGC 6475 (Villanova et al. 2009).

In $\S 6.2$, we describe a framework for predicting the evolution of the upper and lower envelopes of the Li dispersion in young systems. As a test of our models, we compare their predictions with the Li patterns of a number of young clusters and associations. These clusters are NGC 2264, $\beta$ Pictoris, IC 2602, NGC $2451 \mathrm{~A}+\mathrm{B}, \alpha$ Persei, and Blanco 1. Effective temperatures and $\mathrm{A}(\mathrm{Li}) \mathrm{s}$ were taken directly from the literature for these clusters. Ages and $[\mathrm{Fe} / \mathrm{H}] \mathrm{s}$ were obtained from various sources, for calculating their respective model predictions. These sources are listed in Table 2. NGC 2451 A and NGC $2451 \mathrm{~B}$ are two different clusters along the same line of sight, but since they appear to have similar ages and compositions, we combine their data into a single set. Each age comes from the LDB technique, except that of NGC $2451 \mathrm{~A}+\mathrm{B}$, which comes from fitting isochrones to the MS turn-off, and that of NGC 2264. The age of NGC 2264 is a contentious topic; previous studies place it between 0.1 Myr and 10 Myr (see Dahm 2008 for a thorough discussion), but most authors agree there is a substantial age spread within the cluster population. We adopt the age of $6 \pm 3 \mathrm{Myr}$, to roughly bracket the range of literature ages. Each quoted Fe abundance was measured with high-resolution spectroscopy, though we caution that they were not derived uniformly. Furthermore, each author employed their own methodology for 

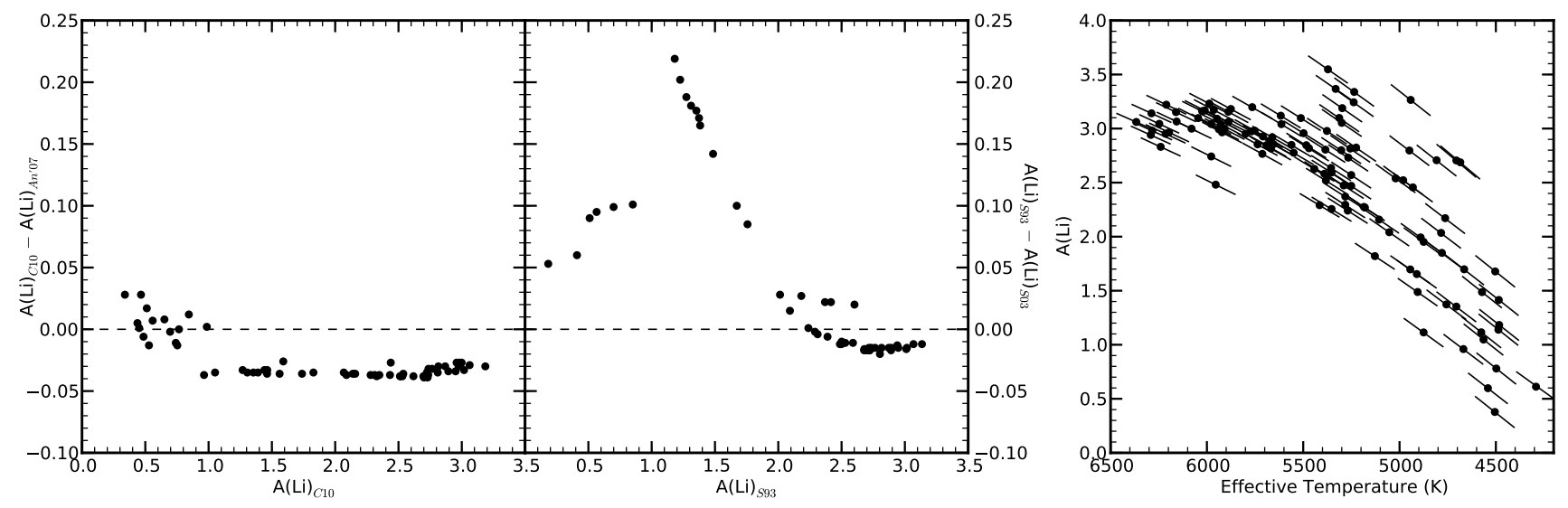

FIG. 2.- Graphic illustrations of the potential systematic errors in our Li abundance analysis (see $\S 2.2$ for details). Left: Hyades abundances derived with the Casagrande et al. (2010) $T_{\text {eff }}$ scale versus the offset between that sample and Hyades abundances derived with the An et al. (2007) $T_{\text {eff }}$ scale. Both use S93 curves of growth. Center: Hyades abundances derived with the S93 curves of growth versus the offset between that sample and Hyades abundances derived with the S03 curves of growth. Both use Casagrande et al. (2010) $T_{\text {eff }}$ scale. Right: The effect of $T_{\text {eff }}$ errors on derived abundances. The line shows the direction and magnitude of data movement resulting from $\pm 100 \mathrm{~K}$ errors in the data. The correlation between $T_{\text {eff }}$ and $\mathrm{A}(\mathrm{Li})$ causes the points to move diagonally, largely preserving the LDP shape in the cool star regime.

deriving effective temperatures and Li abundances. We consider this level of precision acceptable, since these clusters will be used to seek qualitative agreement rather than quantitative rigor.

\subsection{Abundance Analysis}

For each benchmark cluster, we drew $\lambda 6707.8 \mathrm{Li}$ I EWs, and photometric BV measurements, from various literature sources (see Table 1). The Pleiades EWs and photometry come from S93. Hyades EWs come from Thorburn et al. (1993) and Hyades photometry comes from Johnson \& Knuckles (1955). M67 EWs come from Pasquini et al. (2008) and M67 photometry comes from Montgomery et al. (1993). To maximize the internal consistency of our data sets, we did not merge multiple catalogs of Li EWs or photometry. We applied the reddening corrections referenced in Table 1 to these data, calculated effective temperatures using the BV polynomial fit of Casagrande et al. (2010; C10 hereafter), and derived $\mathrm{A}(\mathrm{Li}) \mathrm{s}$ with the curves of growth (CoG) of S93. These CoG are valid between $4000 \mathrm{~K}$, where total depletion on the pre-MS occurs, and $6500 \mathrm{~K}$, where significant additional non-standard mixing occurs on short time scales (the lithium dip - Boesgaard \& Tripicco 1986; Balachandran 1995), so we discard stars that lie outside these bounds. This does not affect our conclusions, as our main concern is solar analogs.

The Li absorption line suffers from blending with a nearby Fe I line located at $6707.4 \AA$. Although the resolution of the Hyades spectra of Thorburn et al. (1993) was high enough to directly remove the blend, the resolution of the Pleiades and M67 spectra was not. The authors therefore removed the blend contribution using the method suggested by S93: the Fe I contribution is calculated as $\operatorname{EW}(\lambda 6707.4$ Fe $\mathrm{I})=20(B-V)-3 \mathrm{~m} \AA$, and subtracted from the measured EW. They estimate that this relation is accurate to $3-5 \mathrm{~m} \AA$, significantly smaller than the errors on the Pleiades EWs, but comparable to the M67 EWs. This may impact the inferred abundances of stars with low EWs, for which blends are naturally harder to remove. However, the $[\mathrm{Fe} / \mathrm{H}]$ of $\mathrm{M} 67$ is similar to the cluster this relation was calibrated on (the Pleiades), so the errors are likely on the low end of the range. Furthermore, systematic offsets should not affect comparisons of Pleiades and M67 stars, given that their EWs were obtained with the same deblending process. Finally, this may introduce minor systematic errors between these data and the Hyades sample (see Thorburn et al. 1993 for a discussion).

To estimate the accuracy of these abundances, we compare Hyades $\mathrm{A}(\mathrm{Li}) \mathrm{s}$ derived from the C10 $T_{\text {eff }}$ scale to abundances derived using temperatures obtained with the An et al. (2007) $T_{\text {eff }}$ scale. This comparison is shown in the left panel of Fig. 2. Both derivations used the CoG of $\mathrm{S} 93$, to isolate the effect of $T_{\text {eff }}$ systematics on our final results. The abundances agreed to better than 0.05 dex for all stars. Errors in $T_{\text {eff }}$ do not have a strong impact on LDPs, because $T_{\text {eff }}$ and $\mathrm{A}(\mathrm{Li})$ are correlated such that $T_{\text {eff }}$ errors move stars diagonally along the pattern (Fig. 2 ; right panel). Though systematic offsets may affect the relative amounts of stars in each $T_{\text {eff }}$ and $\mathrm{A}(\mathrm{Li})$ bin, the median of the pattern is largely unaffected. Next, we combine the Hyades $T_{\text {eff }}$ and $\mathrm{Li}$ EWs presented in Steinhauer (2003; S03 hereafter) with the S93 CoG, and compared the resulting abundances to those presented by S03, who used his own CoG. This is illustrated in the center panel of Fig. 2. There is good agreement for stars with $\mathrm{A}(\mathrm{Li}) \gtrsim 2$, but the derived abundances for Li-poor stars differ by up to 0.22 dex between the CoGs. This is a potentially significant systematic error source, so we perform the analysis with both CoGs and compare the results in $\S 5.1$.

Finally, we compare the derived data for each cluster to alternative abundances patterns from the literature. This is shown in Fig. 3. The black points represent the data we use in this paper, as described above, and the red points represent temperatures and abundances taken directly from literature sources. The comparison data were drawn from Margheim (2007) for the Pleiades, Takeda et al. (2013) for the Hyades, and SR05 for M67. The first two authors derived Li parameters from new spectroscopy of their samples, and SR05 used the M67 Li I EWs of Jones et al. (1999), a distinct data set from the 


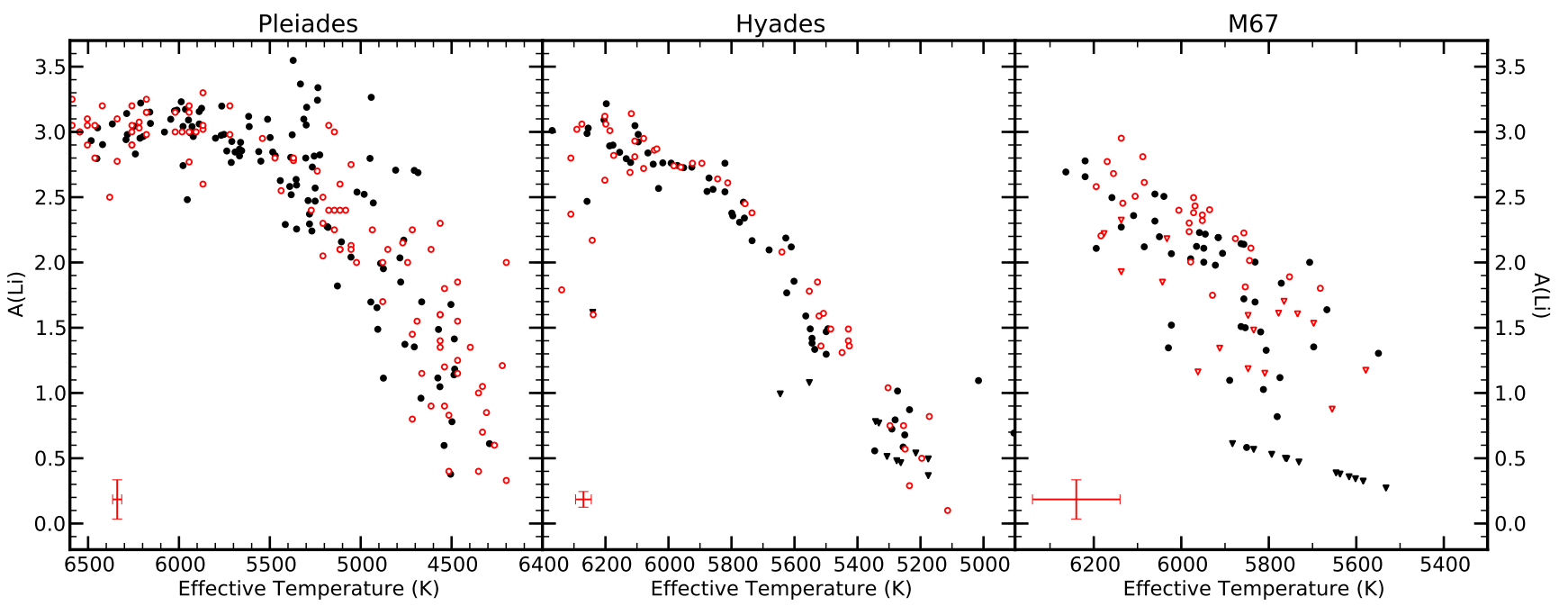

Fig. 3. - A comparison of our benchmark data to alternative data set choices from the literature. Our adopted data, as described in $\S 2.1-2.2$, are shown in filled black. The alternative choices are in empty red: Margheim (2007) for the Pleiades, Takeda et al. (2013) for the Hyades, and Jones et al. (1999) for M67. Typical error bars for the comparison samples are shown in the bottom left.

one employed in our analysis. This figure demonstrates a key point: a consistent analysis method is crucial for controlling systematic effects. Non-uniform parameter derivation leads to systematic offsets between samples; for example, our data is shifted, on average, blue-ward compared to the alternative samples for the Pleiades and the Hyades, and red-ward compared to the alternative M67. However, when placed on the same temperature scale, and the same CoG employed, much of this systematic jitter is removed. Ultimately, our Pleiades and Hyades sets are quite similar to the alternative choices, particularly in the $5500-6100 \mathrm{~K}$ range, where we desire the cleanest sample $(\S 4)$. Our data is more dissimilar with M67, but this is largely due to the improved data quality of Pasquini et al. (2008) relative to that of Jones et al. (1999). Some detections in this sample are for lower abundances than the strictest upper limits from SR05, so we believe that our chosen sample is superior.

\subsection{Stellar Models}

To calculate the SSM predictions of the LDPs of open clusters, we use the Yale Rotating Evolution Code (see Pinsonneault et al. 1989 for a discussion of the mechanics of the code). We adopt a Grevesse \& Sauval (1998) proto-solar metal abundance $(Z / X=0.025293$; this is larger than the current solar surface abundance $Z / X=$ 0.02292 due to gravitational settling), and choose the solar hydrogen mass fraction and the mixing length $(\alpha)$ such that a solar mass model reproduces the solar luminosity and radius at $4.57 \mathrm{Gyr}$. The calibrated values are $\alpha=1.88269, X=0.71304$, the helium mass fraction $Y=0.26882$, and metal mass fraction $Z=0.018035$ for $[\mathrm{Fe} / \mathrm{H}]=0.0$. Our models use the $2006 \mathrm{OPAL}$ equation of state (Rogers et al. 1996, Rogers \& Nayfonov 2002), atmospheric initial conditions from Kurucz (1979), high temperature opacities from the opacity project (Mendoza et al. 2007), low temperature opacities from Ferguson et al. (2005), and the ${ }^{7} \operatorname{Li}(p, \alpha) \alpha$ cross section of Lamia et al. (2012). In each of our models, we assume a initial $\mathrm{Li}$ abundance equal to the proto-solar abundance: $\mathrm{A}(\mathrm{Li})$ $=3.31$ (Anders \& Grevesse 1989). We return to this assumption when evaluating the accuracy of our measure- ments, but for now mention that because Li depletion is logarithmic, $\mathrm{dA}(\mathrm{Li}) / \mathrm{dt}$ does not depend on the abundance.

To obtain the chemical mixture for arbitrary $[\mathrm{Fe} / \mathrm{H}]$, we adopt a big bang helium mass fraction $Y_{B B}=0.2484$ (Cyburt et al. 2004), and assume a linear evolution of $Y$ with metals:

$$
Y=Y_{B B}+\frac{\Delta Y}{\Delta Z} Z
$$

$\Delta Y / \Delta Z$ is solved for by calculating the slope between the big bang mixture $(Y, Z)=(0.2484,0.0)$, and our calibrated solar mixture $(Y, Z)=(0.26882,0.018035)$. We derive $\Delta Y / \Delta Z=1.13$, consistent with recent estimates (Casagrande et al. 2007). We use this method to create abundance mixtures for the adopted $[\mathrm{Fe} / \mathrm{H}]$ of the clusters in this study, and evolve forward models of mass $0.5-1.3 M_{\odot}$, in steps of $0.05 M_{\odot}$, for each.

\section{SOURCES OF ERROR}

We first consider sources of error that could affect the accuracy of our ZAMS Li predictions. These fall into two categories: errors affecting the relative predictions between cluster LDPs, and errors affecting the absolute predictions of all cluster LDPs. The relative error budget is dominated by uncertainties in cluster $[\mathrm{Fe} / \mathrm{H}]$, which shift the predicted LDPs of clusters relative to one another, impacting the inferred lithium anomaly (§3.1). This error has been minimized in our analysis through the selection of clusters with exquisitely measured $[\mathrm{Fe} / \mathrm{H}]$. Absolute errors are dominated by uncertainties in the physics adopted in our models, the physics of Li burning, and the proto-solar abundance, which affect the LDP predictions of all clusters simultaneously. Although these uncertainties are systematic, they can impact the relative predictions of clusters, and thus the inferred anomaly. We describe the effect of these errors in $\S 3.2$, and account for their impact in $\S 4$.

\subsection{Metallicity Errors}

ZAMS LDPs are extremely sensitive to composition. This is demonstrated by Fig. 4, which shows several 


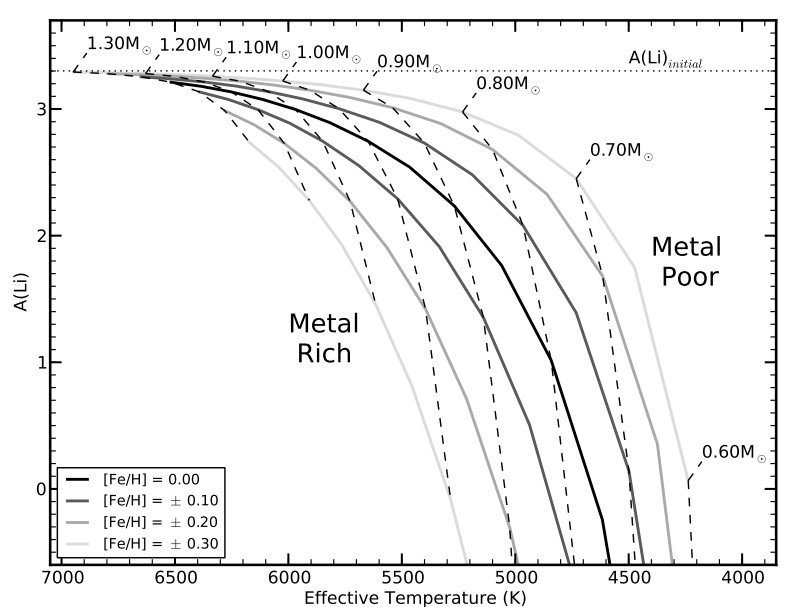

FIG. 4.- SSM Li predictions at the ZAMS for a variety of metallicities, which illustrate the strong dependence of pre-MS Li depletion on composition. The lowest metallicity pattern is the topmost curve, showing that increased metallicity drastically increases the $\mathrm{Li}$ depletion rate at early stages of stellar evolution. X and Z values are calculated with respect to a Grevesse \& Sauval (1998) proto-solar abundance. Dashed lines display curves of constant mass, and the dotted line represents the initial lithium abundance.

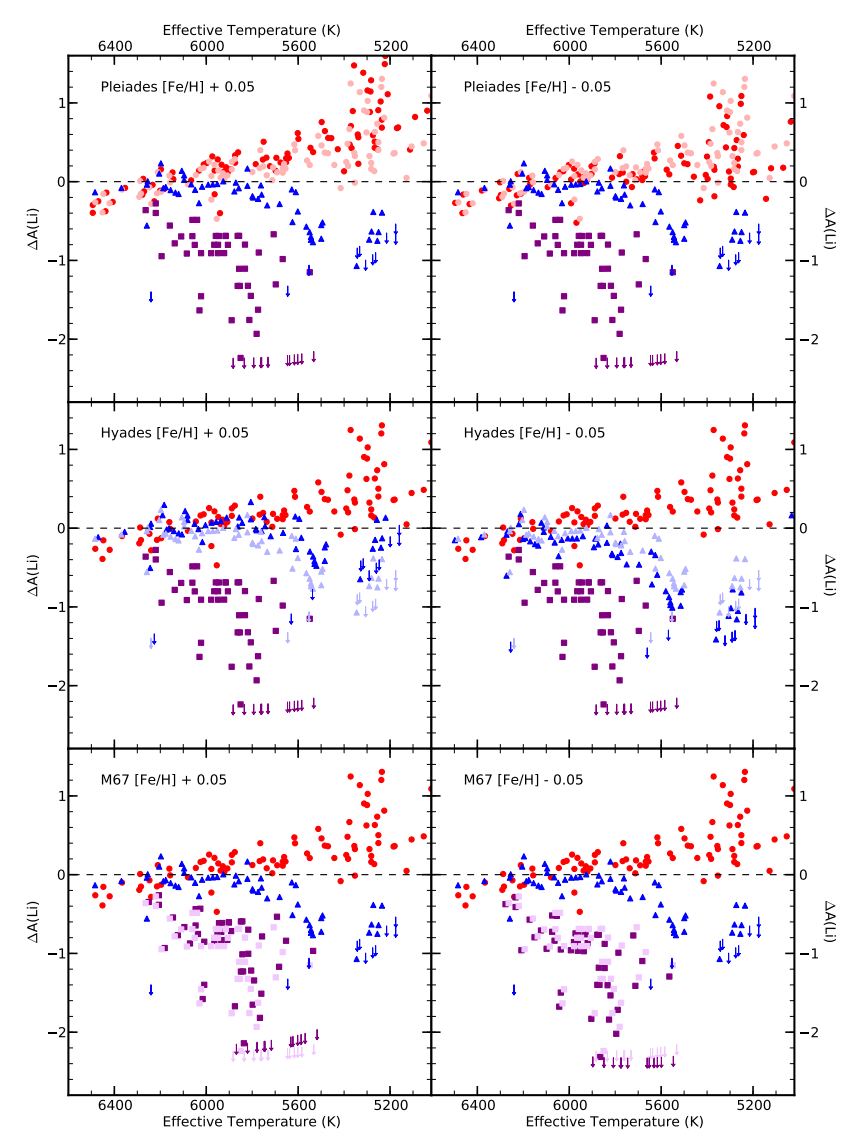

FIG. 5.- The impact of metallicity errors on the relative locations of cluster $\mathrm{Li}$ patterns in $\mathrm{Li}$ anomaly space. In the top row, the transparent red circles represent the anomaly inferred with with original $[\mathrm{Fe} / \mathrm{H}]$, given in Table 1 , and the solid red points are Pleiades anomalies inferred with a different assumed $[\mathrm{Fe} / \mathrm{H}]$, as shown in the top left of each panel. The pattern is shifted significantly if an erroneous composition is assumed. The blue and purple points represent Hyades and M67 anomaly data. The middle and bottom rows repeat the same exercise for the Hyades and M67 respectively. Arrows denote upper limits.
Pleiades-age patterns calculated with a range of $[\mathrm{Fe} / \mathrm{H}] \mathrm{s}$. As can be seen, the abundances vary greatly at fixed $T_{\text {eff }}$ and fixed mass (dashed lines) depending on the composition. More metal-rich clusters are progressively more depleted, and in some cases, a deviation of 0.1 dex in $[\mathrm{Fe} / \mathrm{H}]$ produces a deviation $\gtrsim 1$ dex in $\mathrm{A}(\mathrm{Li})$ ! This strong dependence results from a deeper $\mathrm{CZ}$ in metal-rich stars, which increases $T_{B C Z}$ and drastically increases the burning rate. By contrast, the rate of depletion on the MS is insensitive to composition. Regardless of metallicity, $T_{B C Z}$ on the MS is much less than $T_{L B}$, suggesting that the rate of $\mathrm{Li}$ destruction cannot depend on the thermal properties of the envelope. Therefore, once the $[\mathrm{Fe} / \mathrm{H}]$-dependent pre-MS signal is removed, comparisons between MS LDPs become stable to metallicity errors.

The sensitivity of the benchmark cluster anomalies on the adopted $[\mathrm{Fe} / \mathrm{H}]$ is seen in Fig. 5. Here, we show the cluster data subtracted from a variety of SSM LDPs. In the top left and right, the solid red circles show the distribution of stars had we assumed an $[\mathrm{Fe} / \mathrm{H}]$ for the Pleiades that was 0.05 dex higher or lower, respectively. The ghosted red circles show the pattern produced by assuming the adopted $[\mathrm{Fe} / \mathrm{H}]$ in Table 1 , and the Hyades (blue triangles) and M67 (purple squares) are the same as in the bottom row of Fig. 1. The middle row shows the same effect but for the Hyades, and the bottom row for M67. Fig. 5 demonstrates that metallicity errors move detrended cluster LDPs relative to one another. A very small logarithmic shift in $[\mathrm{Fe} / \mathrm{H}]$ can introduce a large fractional shift between two detrended clusters, significantly altering the inferred magnitude of MS depletion. The formal $[\mathrm{Fe} / \mathrm{H}]$ error for each of our benchmark clusters is less than 0.05 dex, so composition-related errors are well controlled in our measurements, but these effects will be important when comparing clusters with less precisely determined relative metallicities. Furthermore, metallicity errors are far more important for low-mass stars. This suggests higher-mass stars are more stable to $[\mathrm{Fe} / \mathrm{H}]$ uncertainties.

\subsection{Theoretical Systematics}

Lithium depletion is extremely sensitive to the adopted stellar physics (D'Antona \& Mazzitelli 1994; Piau \& Turck-Chièze 2002; Tognelli et al. 2012). There are several physical inputs that could potentially impact Li predictions, the most important of which we list in Table 3. To estimate the theoretical errors associated with these components on the pre-MS, we adopted the SSM prediction for the Pleiades as a fiducial model. We then varied each source of uncertainty in turn, computed the resulting LDP, and compared it to our fiducial pattern. The results can be seen in the left column of Fig. 6. The top panel shows the differences between the fiducial pattern and each alternative pattern as function of $T_{\text {eff }}$, and the bottom panel shows the quadrature sum of the uncertainties, with the width of the error band enumerated at periodic intervals. We treat the sum of systematic differences between inputs, such as distinct equation of state tables or opacity calculations, as effective $2 \sigma$ errors.

Our theoretical errors are asymmetric. This is largely because changes in the solar heavy element mixture systematically reduce Li burning; adopting the Asplund et al. (2009) abundance ratios has an effect analogous to reducing $[\mathrm{Fe} / \mathrm{H}]$ by $\sim 0.1$ dex. The equation of state is 


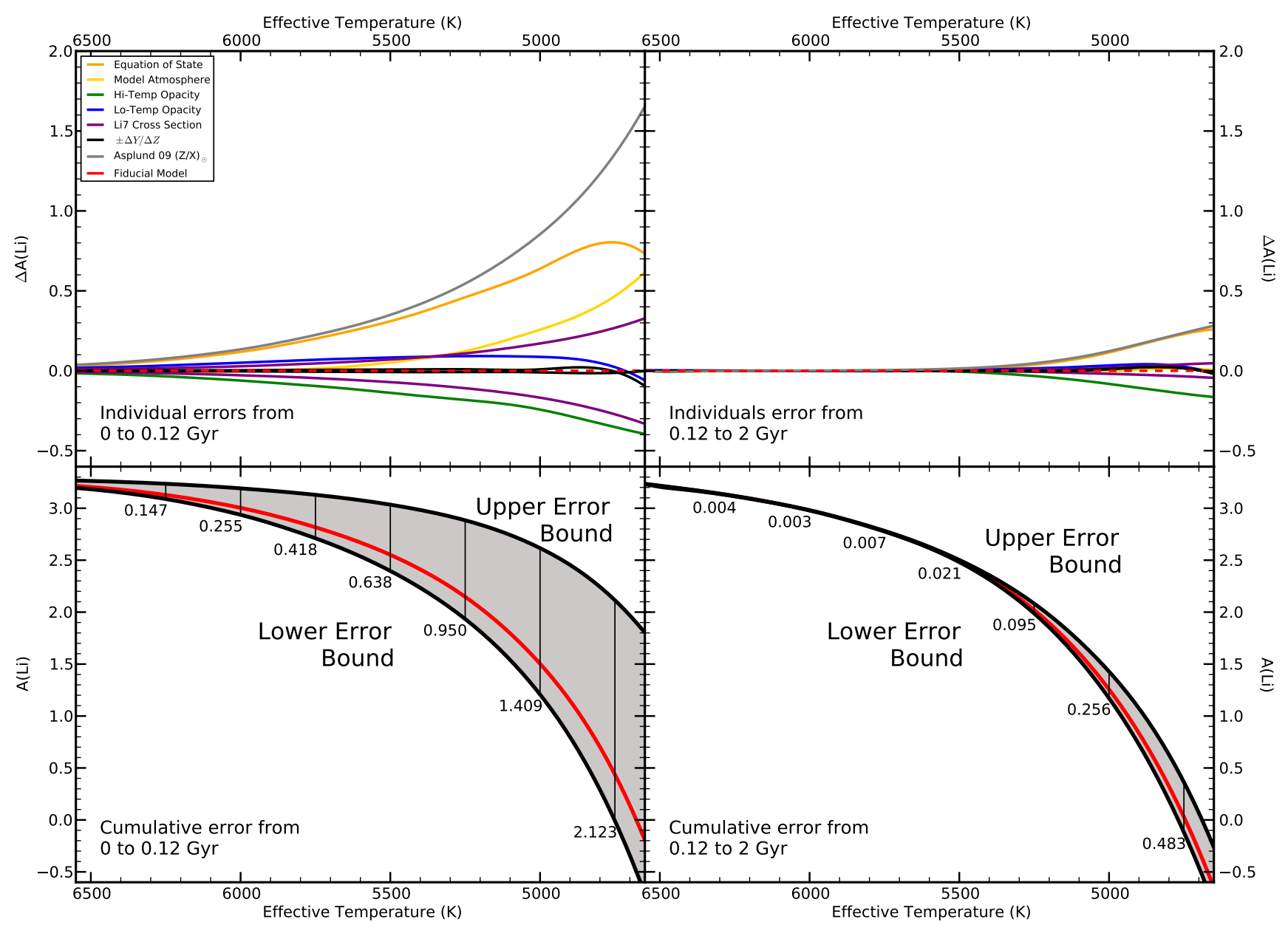

FIG. 6.- The Li depletion errors induced by uncertainties in the adopted model during the pre-MS (left), and during the MS (right). Top: The difference between the fiducial Li pattern and the Li pattern resulting from exchanging one physical input for another commonly adopted in the literature. Colors correspond to different physical inputs, as enumerated in the key. The errors in the left panel develop during the first $0.12 \mathrm{Gyr}$, and those on the right develop between 0.12 and 2 Gyr. Depletion on the MS is insensitive to physical errors, Bottom: The quadrature sum of the errors as a function of $T_{\text {eff }}$. Any Li curve lying in the shaded region is a statistically acceptable Li depletion pattern.

the second largest effect, because a different relationship between $T$ and $p$ can change the depth of the surface convection zone $(\mathrm{CZ})$, altering the temperature at its base and the rate of Li destruction. Other significant effects include the choice of model atmosphere, though this is a large effect only for stars with MS $T_{\text {eff }}<5000 \mathrm{~K}$, and the ${ }^{7} \operatorname{Li}(p, \alpha) \alpha$ cross-section, which produces symmetrical LDPs about the fiducial choice. There is a larger dynamic range in the model uncertainties for cool stars than for hot stars, similar to errors induced by metallicity uncertainties, because of their lengthier pre-MS burning phase.

We repeated the above exercise to investigate the impact of theoretical uncertainties on the minor SSM depletion occurring on the MS. To do this, we evolved our fiducial and alternate stellar models from the age of the Pleiades to 2 Gyrs, and measured the additional discrepancy that develops during this time period. This can be see in the right column of Fig. 6. The impact on hot stars is almost nonexistent. The base of the $\mathrm{CZ}$ above $5500 \mathrm{~K}$ is so cool during this period that small changes to the thermal structure of the envelope do not result in substantial changes to the rate of Li destruction. Minor changes are seen in cool stars, whose CZ bases are still somewhat warm, but at a much lower level than the uncertainties developing on the pre-MS. This demonstrates that once a depletion pattern has been corrected for theoretical errors arising on the pre-MS, the inferred MS depletion is stable.

Fig. 7 shows data from our benchmark clusters detrended with respect to the bottom and top of the preMS systematic error band, and the fiducial model. While $[\mathrm{Fe} / \mathrm{H}]$ errors move individual clusters with respect to one another, theoretical uncertainties move the clusters up and down in tandem. Uncertainties inherent to our models are predominately an absolute bias level in the magnitude of Li depletion, rather than a relative error between the clusters. Nevertheless, the inferred difference between clusters can change by a few tenths of a dex at fixed $T_{\text {eff }}$ depending on the choice of systematics, because more metal-rich clusters have a wider systematic error band. For instance, the anomaly measurement at $5800 \mathrm{~K}$ between the Pleiades and Hyades is different in the left and right panels of Fig. 7. This demonstrates the need for guidance in the selection of physical inputs.

\section{CALIBRATING STANDARD STELLAR MODEL PHYSICS}

We have shown that Li depletion is sensitive to a large number of physical inputs. In the absence of compelling 
TABLE 3

Pre-MS Systematic ERrors

\begin{tabular}{|c|c|c|c|c|c|c|c|c|}
\hline Error Source & $\begin{array}{c}\text { Fiducial } \\
\text { Choice }\end{array}$ & $\begin{array}{l}\text { Alternate } \\
\text { Choice }\end{array}$ & $\begin{array}{l}\text { Alternate } \\
\text { Ref. }\end{array}$ & $6500 \mathrm{~K}$ & $6000 \mathrm{~K}$ & $\begin{array}{c}\Delta \mathrm{A}(\mathrm{Li}) \text { at... } \\
5500 \mathrm{~K}\end{array}$ & $5000 \mathrm{~K}$ & $4500 \mathrm{~K}$ \\
\hline $\begin{array}{c}\text { Equation of State } \\
\text { Model Atmosphere } \\
\text { High-Temp Opacity } \\
\text { Low-Temp Opacity } \\
{ }^{7} \mathrm{Li}(p, \alpha) \alpha \text { Cross Section } \\
\text { Initial Solar Composition } \\
\Delta \mathrm{Y} / \Delta \mathrm{Z}\end{array}$ & $\begin{array}{l}\text { OPAL } 2006 \\
\text { Kurucz } \\
\text { OP } 17 \\
\text { Alex } 2006 \\
\text { Lamia } 2012 \\
\text { GS98 } \\
1.13\end{array}$ & $\begin{array}{c}\text { SCV } \\
\text { Allard } \\
\text { OPAL17 } \\
\text { Alex } 1995 \\
\pm 9 \% \\
\text { Asp09 } \\
\pm 0.2\end{array}$ & $\begin{array}{l}(1) \\
(2) \\
(3) \\
(4)\end{array}$ & $\begin{array}{l}+0.03 \\
-0.01 \\
+0.01 \\
-0.03 \\
\pm 0.01 \\
+0.04 \\
\pm 0.00\end{array}$ & $\begin{array}{c}+0.11 \\
-0.02 \\
+0.02 \\
-0.08 \\
\pm 0.03 \\
+0.14 \\
+0.01 /-0.01\end{array}$ & $\begin{array}{c}+0.31 \\
+0.01 \\
+0.03 \\
-0.17 \\
+0.07 /-0.08 \\
+0.37 \\
+0.03 /-0.03\end{array}$ & $\begin{array}{c}+0.64 \\
+0.16 \\
-0.04 \\
-0.32 \\
+0.17 /-0.19 \\
+0.90 \\
+0.09 /-0.08\end{array}$ & $\begin{array}{c}+0.83 \\
+0.71 \\
-0.40 \\
-0.67 \\
+0.45 /-0.52 \\
+2.01 \\
+0.29 /-0.26\end{array}$ \\
\hline
\end{tabular}

NoтE. - Citations are as follow: (1) Saumon et al. (1995); (2) Allard et al. (1997); (3) Iglesias \& Rogers (1996); (4) Alexander \& Ferguson (1994); (5) Asplund et al. (2009)

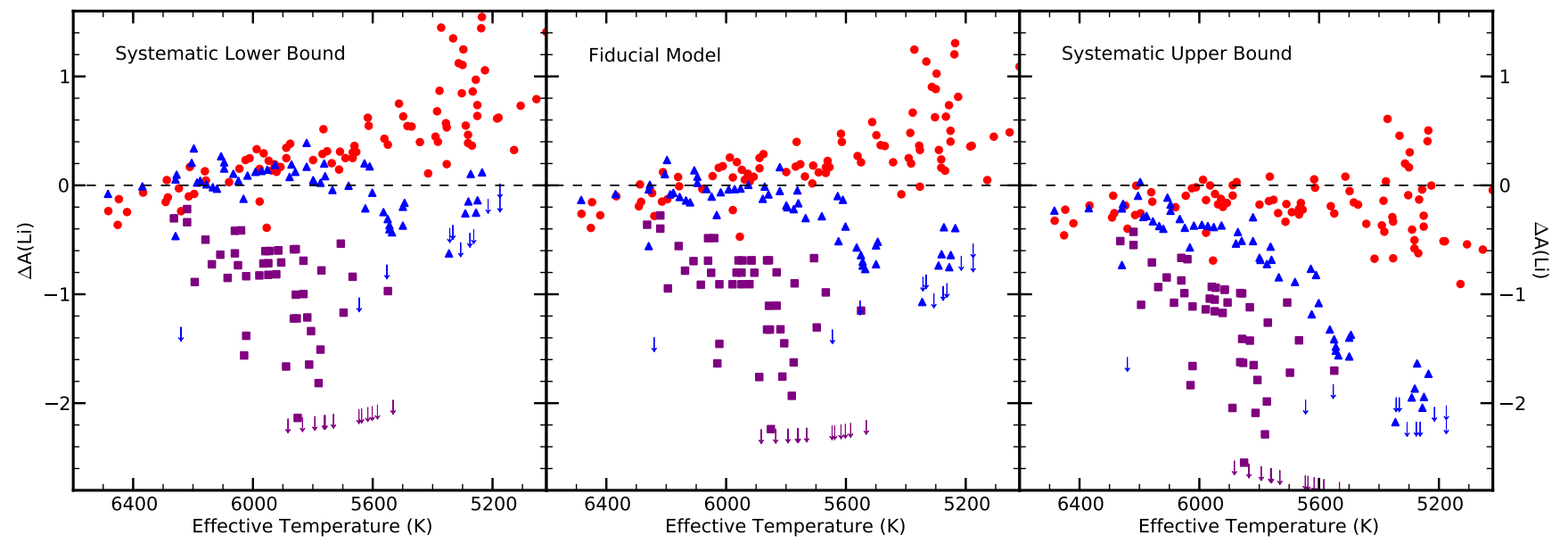

FIG. 7.- The impact of uncertainties in our adopted model physics on the relative locations of clusters in anomaly space. Lithium data for the Pleiades (red circles), Hyades (blue triangles), and M67 (purple squares) are transformed into anomaly space using SSM Li predictions from the bottom envelope of the systematic error band (left), the fiducial model (center), and the top envelope of the systematic error band (right), as illustrated by Fig. 6. The absolute difference between cluster anomaly patterns is sensitive to the adopted model physics, so the models must be calibrated using empirical data. Arrows denote upper limits.

information about which inputs are most in error, we cannot pinpoint which parameter, or parameters, should be changed to reconcile our fiducial theoretical models with nature. However, since MS depletion is insensitive to physics in SSMs, and because there is great theoretical freedom in the ZAMS pattern, it is reasonable to adopt an empirical fit to the absolute depletion of a ZAMS cluster. This gives us an ad hoc calibration of the ensemble physics in our models. Once this has been done for one cluster, SSMs predict the relative scaling required to obtain the calibrated predictions of other clusters. Although this scaling is highly sensitive to errors in metallicity, if the composition of a cluster is reliable, analysis on detrended warm cluster stars will not be biased by significant failures of standard models.

Lithium patterns of young clusters, such as the Pleiades, are expected to closely mimic the true ZAMS LDP, as they have undergone minimal MS depletion. We can therefore use the empirical Pleiades pattern to guide our selection of the theoretical LDP that best reflects the true ZAMS distribution. Before we can do this, we must choose a suitable $T_{\text {eff }}$ range to use for this calibration. As described in $\S 3$, errors induced by both observational and theoretical uncertainties are more significant for cool stars than for hot stars. We therefore restrict our model

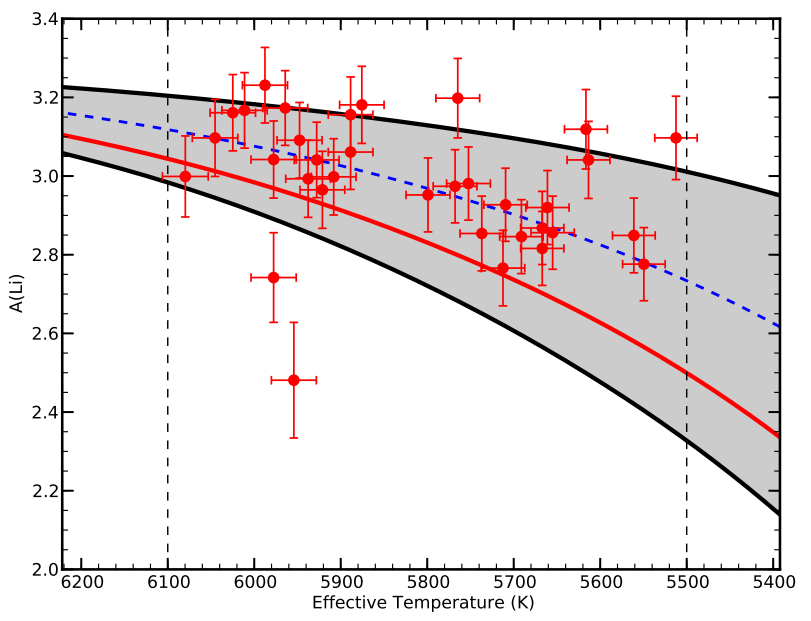

Fig. 8.- Pleiades systematic error band (Fig. 6), over-plotted with Pleiades data in the range 5500K-6100K. Although any LDP lying in the shaded region is statistically acceptable given the uncertainties in our model physics, the dashed blue line represents the best fit to the data. This represents a calibration of the input physics in our models.

calibration to stars with $T_{\text {eff }}>5500 \mathrm{~K}$. At this $T_{\text {eff }}$, the width of the theoretical error band is still large $(\Delta \mathrm{A}(\mathrm{Li})$ $\sim 0.7 \mathrm{dex})$, but the random errors due to uncertainties in 


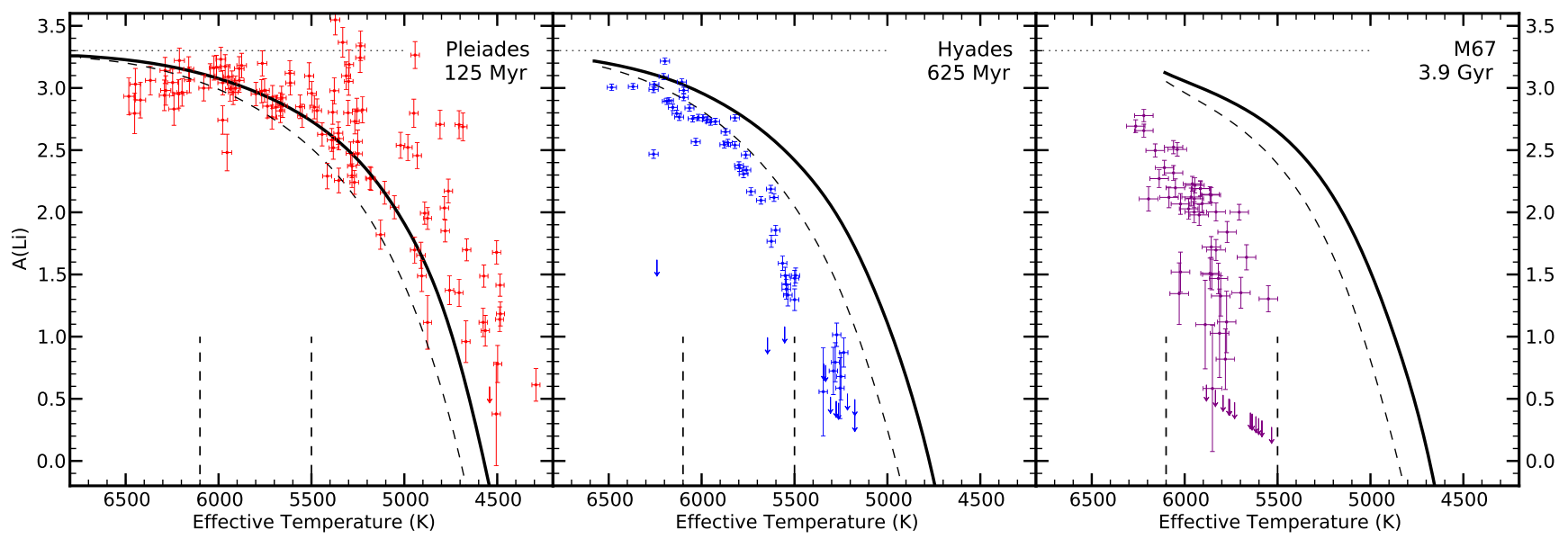

FIG. 9.- Solid black lines represent SSM Li patterns, whose input physics has been calibrated on the Pleiades (§4). Dashed black Li patterns represent the fiducial LDPs. The vertical dashed lines represent the $T_{\text {eff }}$ regime employed to calibrate our model physics. Red, blue and purple data points are the same as in Fig. 1.

$[\mathrm{Fe} / \mathrm{H}]$ are small $(\Delta \mathrm{A}(\mathrm{Li}) \lesssim 0.2$ dex; see Fig. 4). This is beneficial, since we have great leverage in calibrating the input physics due to the large theoretical uncertainties, and relatively small relative errors due to $[\mathrm{Fe} / \mathrm{H}]$ uncertainties. Additionally, we do not include stars with $T_{\text {eff }}$ $>6100 \mathrm{~K}$ in this analysis, because early mixing could be impacting their abundances (Margheim 2007). Our final analysis regime is therefore $5500-6100 \mathrm{~K}$.

We then interpolate to each theoretically allowed ZAMS LDP inside the $2 \sigma$ error bounds in the top left panel of Fig. 6, and determine how well it matches the data by computing the median absolute deviation (MAD) of the empirical Pleiades Li distribution around it. We then select the LDP that produces the best goodness-of-fit within the calibration regime. The resulting function is shown in Fig. 8. Here, the black and red lines are the same as in Fig. 6, and the blue line represents the best fit model. The empirical Pleiades data follow this curve well, with the exception of a few outliers, which may reflect true scatter. We adopt this as our calibrated Pleiades SSM, and as the function we will use to detrend out pre-MS depletion from Pleiades data.

The calibrated patterns for other clusters will lie the same fractional distance between their fiducial LDPs and their upper theoretical error envelopes as the calibrated Pleiades SSM does. To calculate these models, we derive a scale factor from the Pleiades system by dividing the absolute depletion of the detrending function by the absolute depletion of the fiducial model. The scale factor is nearly invariant within the $T_{\text {eff }}$ analysis domain, allowing us to approximate it as a constant. Scaling the Hyades fiducial model by this value produced a close approximation to a detrending function generated by fully calculating the theoretical errors for Hyades parameters and interpolating to the same location as in the Pleiades. This demonstrates that this method can be used to generate systematics-corrected LDPs for any given set of cluster parameters. Our calibrated models for the benchmark clusters are shown as solid black lines in Fig. 9, alongside the fiducial SSMs, which are represented by dashed lines. As can be seen, the tension between the median depletion predicted at ZAMS and solar analogs in the Pleiades has been resolved. This figure also demonstrates that the lower envelope of the cool star dispersion in the Pleiades is well approximated by SSMs. This implies that whatever mechanism is inducing this spread does so by suppressing Li depletion in rapid rotators, and not by inducing additional Li depletion in slow rotators ( $(6.2)$.

We note that the metal content of our models has been determined by scaling the proto-solar abundance by the measured $[\mathrm{Fe} / \mathrm{H}]$ of each cluster. In actuality, $[\mathrm{Fe} / \mathrm{H}] \mathrm{s}$ are reported relative to the solar photospheric abundance. The true metallicity of stars during the pre-MS was larger than their current abundances by a factor equal to the amount of gravitational settling occurring during their lifetime. This is a mass and age dependent effect, in the sense that older and more massive stars undergo more settling. The magnitude of the effect for solar analogs in $\mathrm{M} 67$ is $\Delta[\mathrm{Fe} / \mathrm{H}]=-0.06$, leading to a 0.04 dex change in the predicted pre-MS Li depletion. This effect is smaller for lower mass stars, because settling is negligible when the CZ is deep, and smaller for higher mass stars as well, because the rate of $\mathrm{Li}$ depletion is insensitive to composition in this regime. The corresponding effect is significantly lower for the Hyades, since it is younger by a factor of 8 , and non-existent for the Pleiades, since we have calibrated our models on empirical data for this cluster. Given the complex, and in general unknown, dependence of settling on mass and age, we do not include this effect in our calculations. Given the small magnitude of the effect, it does not significantly impact the accuracy of our measurements.

\section{RESULTS}

Surface Li destruction proceeds rapidly on the pre-MS, and much slower thereafter. To empirically measure the rate of Li depletion on the MS, we must accurately predict the amount of depletion that occurs for stars on the pre-MS, so it can be subtracted from their present day abundances. However, the amount of Li destruction occurring on the pre-MS in SSMs is highly sensitive to errors in both the assumed metallicity of the cluster and the physical inputs in our models. The former can be controlled by considering clusters with well known composition, so we have selected three well-studied clusters for a precision measurement. The latter can be controlled by selecting the input physics which best reproduces the 


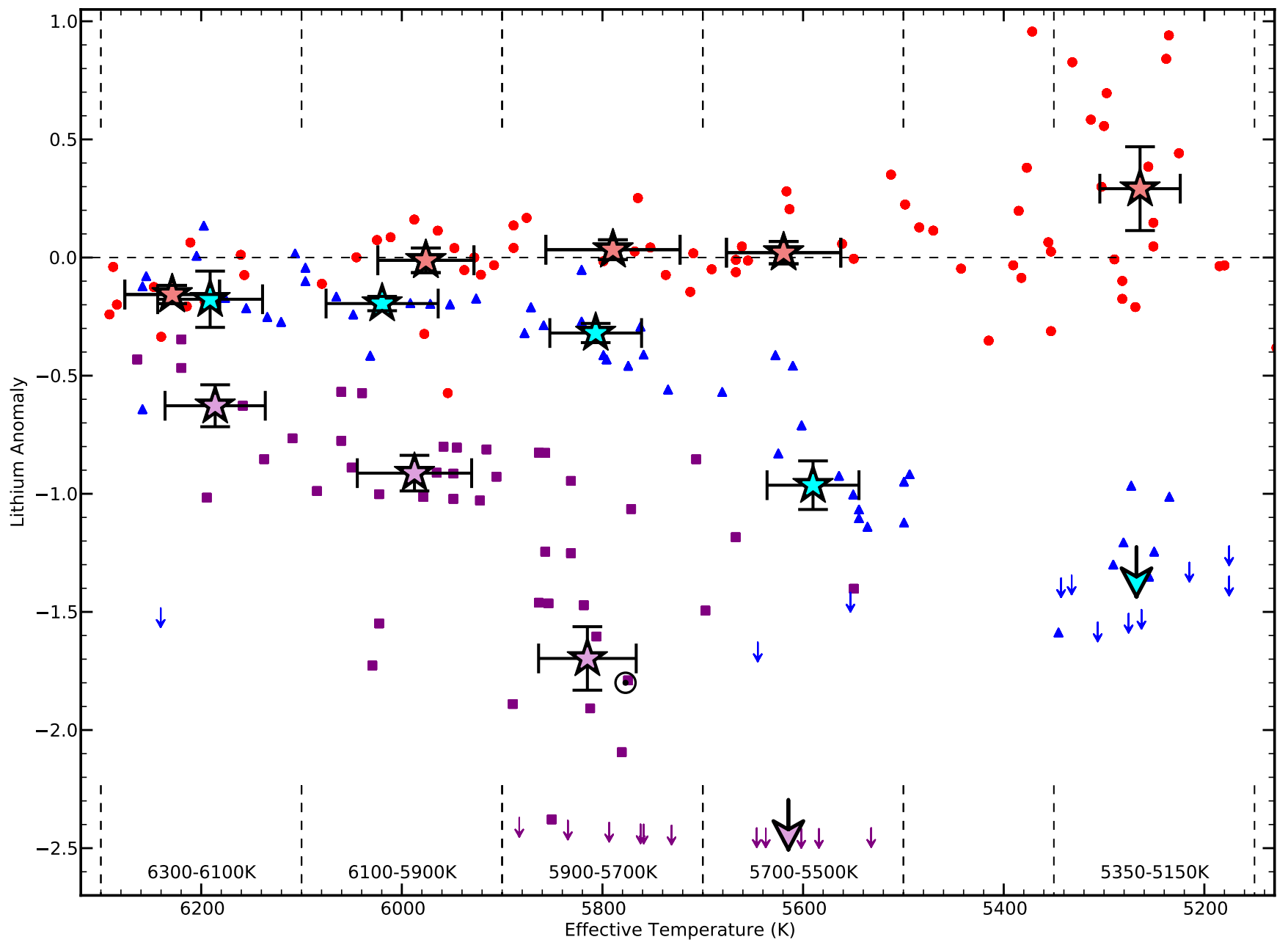

FIG. 10.- The final Li anomaly measurements for our benchmark clusters. The Pleiades (red circles), Hyades (blue triangles) and M67 (purple squares) data have been detrended with respect to their calibrated SSM LDPs, described in $\$ 4$. Light red, light blue, and light purple stars are the average $T_{\text {eff }}$ and median lithium anomaly in each bin (denoted by black dashed lines) for the Pleiades, Hyades and M67, respectively. An inverted triangle denotes an upper limit to the median anomaly. The solar lithium abundance is also shown, and arrows denote upper limits.

observed Li pattern of a ZAMS cluster, which we do in an ensemble fashion in $\S 4$. Once our physics is calibrated, the Li destruction occurring on the pre-MS can be detrended out of an empirical Li pattern, thus isolating the depletion occurring on the MS. The rate of MS Li depletion can then be inferred by comparing the average $\mathrm{Li}$ anomalies of different aged clusters.

In this section, we use this methodology to obtain the MS anomaly for several clusters. First, we will detrend the Hyades and M67, and infer from their anomaly patterns the rate of MS Li depletion, as a function of mass and age. We find a strong mass trend in the rate of MS depletion in both clusters, and confirm that the average rate of depletion decreases at advanced ages. We also discuss a few caveats to this method, including the effects of the initial Li abundances of clusters, and the possibility of cosmic variance differentially impacting cluster LDPs, but conclude that our results are robust. We then detrend and analyze a large sample of open clusters, previously studied by SR05, to explore the timescales of MS depletion and dispersion. We find that the rate of depletion of solar analogs is unchanged by the transformation to anomaly space, but the mass dependence of depletion is stronger in absolute space. Finally, we measure the Li dispersion at fixed $T_{\text {eff }}$ in each cluster, and explore the timescales of its development. We find that dispersion sets in early in many clusters, and can increase, decrease, or remain steady over time depending on the temperature bin. Furthermore, dispersion is not a simple function of age, suggesting cosmic variance in cluster initial conditions.

\subsection{Benchmark Clusters \\ 5.1.1. The Lithium Anomaly}

Our final detrended benchmark clusters are shown in Fig. 10. Here, we have divided our analysis regime into three bins of $200 \mathrm{~K}$ width. This provides us sufficiently low shot noise to calculate the median Li depletion without introducing too much error due to the $T_{\text {eff }}$ dependence of the pattern. We also include two additional $T_{\text {eff }}$ regimes that were excluded in $\S 4$ : $6300-6100 \mathrm{~K}$ and 5350 $5150 \mathrm{~K}$. Although these temperature ranges were not suitable for calibrating our models, the anomaly remains a useful measure of mixing. The light red, light blue, and light purple stars represent the average $T_{\text {eff }}$ and the median lithium anomaly of the Pleiades, Hyades, and M67 within each bin (illustrated by the vertical dashed lines). 
TABLE 4

Benchmark Cluster Lithium Anomalies

\begin{tabular}{|c|c|c|c|c|c|c|}
\hline \multirow{2}{*}{$\begin{array}{l}\text { Curves of } \\
\text { Growth }\end{array}$} & \multirow[t]{2}{*}{ Cluster } & \multicolumn{5}{|c|}{ Anomaly At... } \\
\hline & & $6200 K$ & $6000 \mathrm{~K}$ & $5800 \mathrm{~K}$ & $5600 \mathrm{~K}$ & $5250 \mathrm{~K}$ \\
\hline$S 93$ & $\begin{array}{l}\text { Pleiades } \\
\text { Hyades } \\
\text { M67 }\end{array}$ & $\begin{array}{l}-0.157 \pm 0.039 \\
-0.177 \pm 0.119 \\
-0.627 \pm 0.089\end{array}$ & $\begin{array}{l}-0.012 \pm 0.052 \\
-0.195 \pm 0.030 \\
-0.913 \pm 0.075\end{array}$ & $\begin{array}{c}0.033 \pm 0.042 \\
-0.320 \pm 0.041 \\
-1.697 \pm 0.134\end{array}$ & $\begin{array}{l}0.021 \pm 0.047 \\
-0.964 \pm 0.103 \\
\quad<-2.415\end{array}$ & $\begin{array}{c}0.291 \pm 0.177 \\
<-1.345 \\
-\end{array}$ \\
\hline & $\begin{array}{c}\text { Pleiades } \rightarrow \text { Hyades } \\
\text { Pleiades } \rightarrow \text { M67 } \\
\text { Hyades } \rightarrow \text { M67 }\end{array}$ & $\begin{array}{l}0.020 \pm 0.125 \\
0.471 \pm 0.097 \\
0.451 \pm 0.149\end{array}$ & $\begin{array}{l}0.183 \pm 0.060 \\
0.901 \pm 0.092 \\
0.718 \pm 0.081\end{array}$ & $\begin{array}{l}0.353 \pm 0.058 \\
1.730 \pm 0.141 \\
1.377 \pm 0.140\end{array}$ & $\begin{array}{l}0.984 \pm 0.113 \\
>2.436 \\
>1.451\end{array}$ & $\begin{array}{c}>1.636 \\
- \\
-\end{array}$ \\
\hline $\mathrm{SO} 3$ & $\begin{array}{c}\text { Pleaides } \\
\text { Hyades } \\
\text { M67 }\end{array}$ & $\begin{array}{l}-0.141 \pm 0.038 \\
-0.162 \pm 0.127 \\
-0.623 \pm 0.095\end{array}$ & $\begin{array}{c}0.001 \pm 0.052 \\
-0.177 \pm 0.031 \\
-0.950 \pm 0.083\end{array}$ & $\begin{array}{c}0.039 \pm 0.041 \\
-0.306 \pm 0.042 \\
-1.891 \pm 0.127\end{array}$ & $\begin{array}{c}0.018 \pm 0.046 \\
-1.085 \pm 0.124 \\
<-1.980\end{array}$ & $\begin{array}{c}0.259 \pm 0.174 \\
<-1.549 \\
-\end{array}$ \\
\hline & $\begin{array}{c}\text { Pleaides } \rightarrow \text { Hyades } \\
\text { Pleaides } \rightarrow \mathrm{M} 67 \\
\text { Hyades } \rightarrow \text { M67 }\end{array}$ & $\begin{array}{l}0.021 \pm 0.132 \\
0.482 \pm 0.103 \\
0.461 \pm 0.159\end{array}$ & $\begin{array}{l}0.177 \pm 0.060 \\
0.950 \pm 0.098 \\
0.773 \pm 0.089\end{array}$ & $\begin{array}{l}0.345 \pm 0.059 \\
1.930 \pm 0.133 \\
1.585 \pm 0.133\end{array}$ & $\begin{array}{c}1.103 \pm 0.133 \\
>1.998 \\
>0.895\end{array}$ & $\begin{array}{c}>1.808 \\
- \\
-\end{array}$ \\
\hline
\end{tabular}

Note. - Absolute and relative MS Li depletion factors. The upper values were calculated with the curves of growth of Soderblom et al. (1993a), and the lower values with the curves of growth of Steinhauer (2003).

We have ignored the $T_{\text {eff }}$ range $5500-5350 \mathrm{~K}$, due to a gap in the Hyades data in this regime.

Between the Pleiades and the Hyades, we find the following lithium anomalies, where the first quoted error is due to Poisson noise, and the second is due to $[\mathrm{Fe} / \mathrm{H}]$ uncertainties: $0.020 \pm 0.124 \pm 0.018 \mathrm{dex}$ at $6200 \mathrm{~K}, 0.183$ $\pm 0.057 \pm 0.018 \mathrm{dex}$ at $6000 \mathrm{~K}, 0.353 \pm 0.055 \pm 0.025$ dex at $5800 \mathrm{~K}, 0.984 \pm 0.111 \pm 0.021 \mathrm{dex}$ at $5600 \mathrm{~K}$, and $\gtrsim 1.636$ dex at $5250 \mathrm{~K}$. Between the Pleiades and M67, we find anomalies of $0.471 \pm 0.094 \pm 0.025$ at $6200 \mathrm{~K}$, $0.901 \pm 0.082 \pm 0.041 \mathrm{dex}$ at $6000 \mathrm{~K}, 1.730 \pm 0.128 \pm$ $0.058 \mathrm{dex}$ at $5800 \mathrm{~K}$, and $\gtrsim 2.436 \mathrm{dex}$ at $5600 \mathrm{~K}$. There is no anomaly for the $5250 \mathrm{~K}$ bin here, since no $\mathrm{M} 67 \mathrm{Li}$ data exists in the literature in this range. The Hyades anomaly at $5250 \mathrm{~K}$ and the M67 anomaly at $5600 \mathrm{~K}$ are upper limits, due to the preponderance of upper limits in this bin. These measurements are collected in Table 4. Our choice of benchmarks with well-constrained $[\mathrm{Fe} / \mathrm{H}]$ has caused the uncertainties to be dominated by shot noise.

In $\S 2.2$, we compared the S93 CoG used in this paper with the S03 CoG, and found substantial differences in final abundance for some stars. To evaluate the impact of this uncertainty on our final answer, we compare our answers to anomaly values derived using the S03 CoG. These are also shown in Table 4 . For all but the coolest $T_{\text {eff }}$ bin, the relative anomalies between the Pleiades and Hyades agree with our original values at $\sim 1 \sigma$ or better. Between the Pleiades and M67, the values agree at $\sim 1.5 \sigma$ or better. This increases our confidence that our results are robust. The uncertainties are somewhat larger for M67, since fractional errors increase when EWs are small. The lower limits derived with S03 are $\sim 0.2$ dex lower for the $5250 \mathrm{~K}$ bin, reflecting the sensitivity of derived abundances on EWs in Li-poor stars. This error is not too worrisome, as 0.2 dex represents $<15 \%$ of the total anomaly at this temperature.

An important conclusion about MS Li depletion in FGK dwarfs can be drawn from this plot: low mass stars deplete Li more rapidly than high mass stars on the MS. While this effect has been seen previously in absolute space, our work confirms that the effect persists when the additional depletion suffered by low-mass stars on the pre-MS has been removed. This result holds regardless of the choice of theoretical bias and CoG. This provides a stringent constraint on mechanisms seeking to explain the MS lithium anomaly in open clusters. A second conclusion we can draw from this plot is that the average rate of MS depletion is higher for the Hyades than for M67. This can be seen in Fig. 10 by the ratio of the anomaly at the age of M67 to the anomaly at the age of the Hyades. If the depletion rate were constant for these two clusters, this ratio should be $\sim 7.6$, equal to the ratio of time the clusters have spent on the MS. However, in the $5800 \mathrm{~K}$ and $6000 \mathrm{~K}$ bins, the ratio is 4.8 and 5.1 respectively. This depletion plateau has been seen before (i.e. SR05), but we have shown that the result persists even when differential metallicity effects are accounted for.

These arguments are strengthened by the cosmic evolution of lithium. The Li content of the interstellar medium has increased over time (Spite \& Spite 1982), and a correlation between cluster Fe abundance and initial Li abundance has also been reported (Cummings 2011), so it is possible in principle that our benchmark clusters began their lives with different $\mathrm{Li}$ abundances. The initial M67 Li content is likely close to solar, since it is nearly equal to the Sun in both age and metallicity. The initial Pleiades Li abundance has also been measured to be near solar (e.g. Cummings 2011). However, the Hyades may have been born with a higher $\mathrm{Li}$ abundance than we have assumed. If this is correct, the true magnitude of MS depletion for the Hyades will be greater than we have measured. This would reduce the difference between the Hyades and M67 medians, decrease the ratio described above, and therefore increase the tension between our measurement and the putative expectations of constant logarithmic depletion. Furthermore, changing the assumed initial abundance of a cluster moves each of its members up and down in tandem, and so will not change the mass-dependent pattern revealed through detrending. We therefore believe that shifts in the initial cluster abundance could impact the precision of our anomaly measurements, but will not impact either of the 
conclusions stated above. A rigorous evaluation of the Hyades initial cluster abundances can be undertaken by analyzing the Li content of stars blue-ward of the Li gap, as they will have suffered minimal pre-MS and MS depletion.

It is plausible that additional cluster parameters could impact the Li pattern, jeopardizing the generality of our measured anomalies. For example, rotation is expected to drive deep mixing flows through meridional circulation and shear instabilities, and induce non-standard Li depletion during the MS as a result (e.g. Pinsonneault et al. 1997, and refs. therein). The rate of depletion increases with faster rotation, so clusters with different rotation distributions will eventually develop different LDPs. Environment could therefore impact the Li depletion properties of clusters.

The early (0-10 Myr) rotation evolution of a star is dictated by its circumstellar disk, which locks magnetically to the star and efficiently drains AM from the envelope (e.g. Koenigl 1991; Rebull et al. 2006). If a T Tauri star has a close interaction with another cluster member, its circumstellar disk may be subject to early disruption through the interaction. This will truncate the timescale of the disk-locking phase, and the star will retain the AM it would have otherwise lost, appearing as a rapid rotator at the ZAMS. Such interactions are more likely in dense stellar environments, so this process could induce a correlation between the number density of a cluster at birth, and the fraction of rapidly rotating stars. Assuming a connection between rapid rotation and MS Li depletion, dense clusters would be expected to host a commensurately large fraction of Li-poor stars in the solar regime. This would impact both the width of the Li distribution, and the median anomaly. Although this explanation is qualitatively sensible, Bouvier et al. (1997) found that the rotation rates of primaries in binary systems are statistically indistinguishable from rotation rates of single stars in the Pleiades. This suggests that physics local to the star sets rotation rates, and not environmental factors.

\subsubsection{Literature Comparison}

We now compare our results to SR05, who calculated the average $\mathrm{Li}$ abundance in three $T_{\text {eff }}$ bins at a variety of ages along the MS. It should be noted that there are two important differences between our analysis and that of SR05. First, we measured relative abundances in lithium anomaly space, whereas SR05 worked in absolute abundance space. The practical effect is that SR05 did not correct for differential depletion on the pre-MS due to composition differences. Second, we analyzed only one cluster at a time, whereas SR05 grouped several similarly-aged clusters together and calculated an ensemble average. Their method tends to wash out both random errors in $T_{\text {eff }}$ and $\mathrm{A}(\mathrm{Li})$, and the effect of different metallicities.

With these precautions, we compare results in Fig. 11. In the top panel, the red points are our lithium anomaly measurements for the Hyades, and the blue points represent the difference between the bins containing the Hyades and the bins containing the Pleiades in SR05. The measurements are generally consistent with one another. Between $5700 \mathrm{~K}$ and $6200 \mathrm{~K}$, we measure a marginally smaller Hyades depletion than SR05. This is

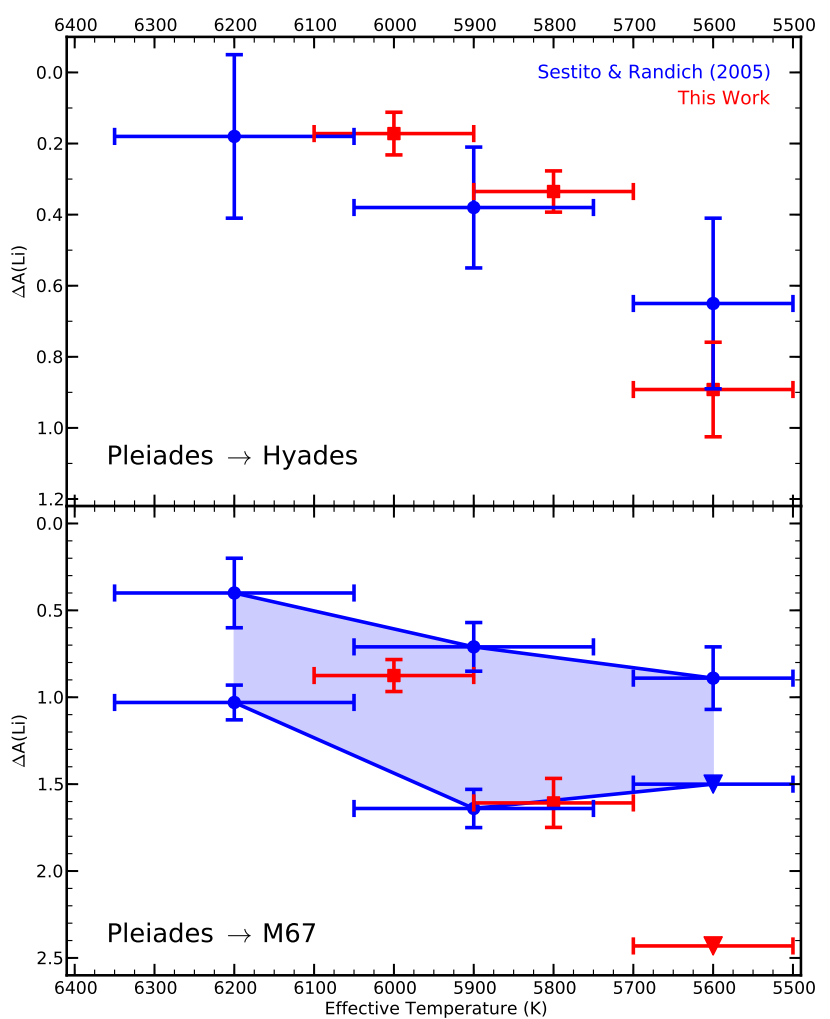

FIG. 11. - A comparison of the lithium anomaly measurements of this work with the measurements of SR05. Red squares represent this work's calculation of the lithium anomaly for the Hyades (top) and M67 (bottom). Blue circles represent the difference between the SR05 bin containing the Pleiades and the bins containing the Hyades (top) and M67 (bottom). SR05 reported the average of the upper and lower envelopes for M67, so we include both sets of points, and show the region interior. Triangles represent upper limits.

because the Hyades is a metal-rich cluster, and thus less MS depletion is inferred when pre-MS effects are considered. However, our measurement is lower than the SR05 measurement at $5600 \mathrm{~K}$. Given the uncertainties inherent to CoG analysis in low abundance regimes, this may reflect differences in the abundance derivation rather than a true difference in measurements. Alternatively, given the steepness of the LDP in this $T_{\text {eff }}$ range, this difference may be due to random errors in our sample. More stars populate the warm side than the cool side of this bin, biasing our answer towards greater depletion.

The red points in the bottom panel of Fig. 11 represent our lithium anomaly measurements for M67. SR05 did not bin M67 with other clusters, but instead measured the average of the M67 upper and lower envelopes separately. We therefore show the range in which the global average of their sample could reside. Our measurement at $6000 \mathrm{~K}$ is in good agreement with SR05. This is not surprising, since M67 and Pleiades are very similar in $[\mathrm{Fe} / \mathrm{H}]$, and thus pre-MS corrections are minimal. Our $5800 \mathrm{~K}$ and $5600 \mathrm{~K}$ measurement are large compared to SR05, but this is likely due to the different data samples used in the two studies. The sample of SR05 is dominated by Li measurements from Jones et al. (1999), which had a lower detection threshold than Pasquini et al. (2008), used by this work. We thus find a lower average at $5800 \mathrm{~K}$, and set a stricter upper limit at $5600 \mathrm{~K}$. 


\subsection{Additional Clusters}

Minimal measurement errors have made the Hyades and M67 optimal for calibrating and testing mixing calculations, but two temporal points do not provide a complete time line of Li evolution. We therefore apply an analysis similar to $§ 5.1$ to the full sample of open clusters described in SR05. They assembled all substantial FGK dwarf Li data sets available prior to 2005, and applied a uniform abundance analysis to examine the timescales of $\mathrm{Li}$ evolution. The results were the confirmation of a mass trend in the rate of MS depletion, and the identification of four stages of depletion: depletion on the preMS, a stall near the ZAMS, depletion on the MS, and a plateau at late ages. Given the considerations put forth in this paper, we wish to determine if these qualitative and quantitative results are altered by the transformation to anomaly space. To this end, we reanalyze with our methods the data described in $\$ 2.1 .2$, excluding the clusters NGC 2264, since substantial pre-MS Li depletion has yet to occur in this young cluster, and NGC 2547 , due to the lack of a quality $[\mathrm{Fe} / \mathrm{H}]$ measurement. We retain the data sets used in $\S 5.1$ for our benchmark clusters.

\subsubsection{Evolution of the Median}

We first compared our results with those of SR05 in absolute space. The data for each cluster were divided into the three $T_{\text {eff }}$ regions they considered $(5600 \pm 100 \mathrm{~K}, 5900$ $\pm 150 \mathrm{~K}$, and $6200 \pm 150 \mathrm{~K})$, and the median of each bin was calculated. We then combined the data from similaraged clusters in the fashion of SR05 (see their Table 3), to maximize the validity of the comparison. Unsurprisingly, our findings are consistent with theirs. A decline in median abundance as a function of age is present in each $T_{\text {eff }}$ bin, and the rate of depletion increases with decreasing mass. Modest differences between our points and theirs are present, due to the difference between mean and median statistics, and our choice of different data sets for some clusters. Nevertheless, this confirms the similarity of the two analysis processes.

Next, we detrended each cluster, using the machinery of $\S 2.3$ and the systematic corrections of $\S 4$, and binned the data as described above. We display each cluster as a single data point instead of applying age bins, allowing for a visual impression of the intrinsic scatter of cluster medians about the mean trend. Finally, we rejected bins with less than 3 members, and plotted the data in the first three panels of the left column of Fig. 12. Black points represent the median anomaly of each cluster in the quoted bins, and the error bars represent the quadrature sum of the standard error of the median $\left(\frac{\mathrm{MAD}}{\sqrt{N}}\right)$, and the uncertainty of the median due to $[\mathrm{Fe} / \mathrm{H}]$ errors. We also plot the data of SR05 in red, with the relevant scale displayed on the right axis. Finally, we binned and detrended data in the $T_{\text {eff }}$ range $5300 \pm 100 \mathrm{~K}$, and plotted the data in the bottom left panel.

As evinced by this figure, cooler stars possess a higher average depletion rate over their lifetimes in both absolute and anomaly space, in agreement with the findings of $§ 5.1$ and SR05. In order to quantify this effect, we calculate the best fit power law for the data in each bin up to 2 Gyr, when the subdivision of M67 in the SR05 data and upper limits begin to complicate the regres- sion process. The red line in each panel shows the best fit for the SR05 data, and the slope is reported in the lower left corner. The $6200 \mathrm{~K}$ bin depletes slower than the $5900 \mathrm{~K}$ bin by a factor of 1.9 , and slower than the $5600 \mathrm{~K}$ bin by a factor of 2.9 . This trend is preserved in anomaly space, but the mass dependence is found to be marginally steeper: depletion in the the detrended $6200 \mathrm{~K}$ bin is consistent with the absolute $6200 \mathrm{~K}$ bin, but is slower by a factor of 2.4 compared to the $5900 \mathrm{~K}$ bin, and slower by a factor of 4.4 compared to the $5600 \mathrm{~K}$ bin. The bottom left panel of Fig. 12 shows that this trend continues beyond the $T_{\text {eff }}$ range considered by SR05; the depletion rate at $5300 \mathrm{~K}$ is a factor of 6.2 greater than in the warmest bin. The anomalies up to $\sim 150 \mathrm{Myr}$ are positive in this bin, reflecting the over-abundance of cool stars relative to theory (see $\S 6.2$ ), and drop sharply after this age. Therefore, the rate of MS depletion after this age is likely higher than reflected by the best fit power law slope.

SR05 argued that abundances in each $T_{\text {eff }}$ bin converge to a plateau after about 2 Gyr. However, this late-time plateau behavior does not satisfactorily describe the median of M67. While the upper envelope of M67 shares the same average abundance as the 2 Gyr and 8 Gyr old clusters in the SR05 data, the large dispersion present in this cluster causes the median abundance to be lower by several tenths of a dex. This can clearly be seen in our data in the $5900 \mathrm{~K}$ bin, where the median abundance at 5 Gyr is substantially less than the older and younger clusters. This suggests that M67 may represent a different evolutionary pathway for cluster Li patterns than the 2 and 8 Gyr old clusters considered here, and points to cosmic variance affecting cluster evolution. In order to quantify the extent of this variance, we now examine the range of Li dispersions that can develop in open clusters.

\subsubsection{Evolution of the Dispersion}

Some proposed MS mixing mechanisms naturally predict a range of depletion rates at fixed mass, and thus a variable dispersion as a function of time (i.e. rotational mixing), while others predict that all stars of a given mass deplete $\mathrm{Li}$ at equal rates on the MS, implying a fixed dispersion (i.e. gravity wave mixing). The evolution of the distribution of $\mathrm{Li}$ abundances at fixed $T_{\text {eff }}$ therefore provides an additional constraint on MS mixing. This quantity is difficult to measure in absolute space, as strong mass trends impart a large difference in average $\mathrm{A}(\mathrm{Li})$ at the high and low ends of cool $T_{\text {eff }}$ bins. This introduces significant scatter about the median abundance of the bin, rendering this measurement impossible without excellent number statistics. However, this issue can be resolved by transforming the data to $\mathrm{Li}$ anomaly space. In this plane, the intrinsic scatter due to the presence of pre-MS mass trends is removed, and a robust measurement of dispersion is possible.

To this end, we calculated the standard deviation of each $T_{\text {eff }}$ bin from $\$ 5.2 .1$, and plot the results in the right column of Fig. 12. The black circles represent the measured dispersions, and the diameter of the circle reflects the sample size: the smallest circles have 3 members, and the largest has 34 . There are three potential sources of noise in the dispersion measurement. The first results from random $T_{\text {eff }}$ errors, which can cause individual stars to be detrended improperly, altering the 

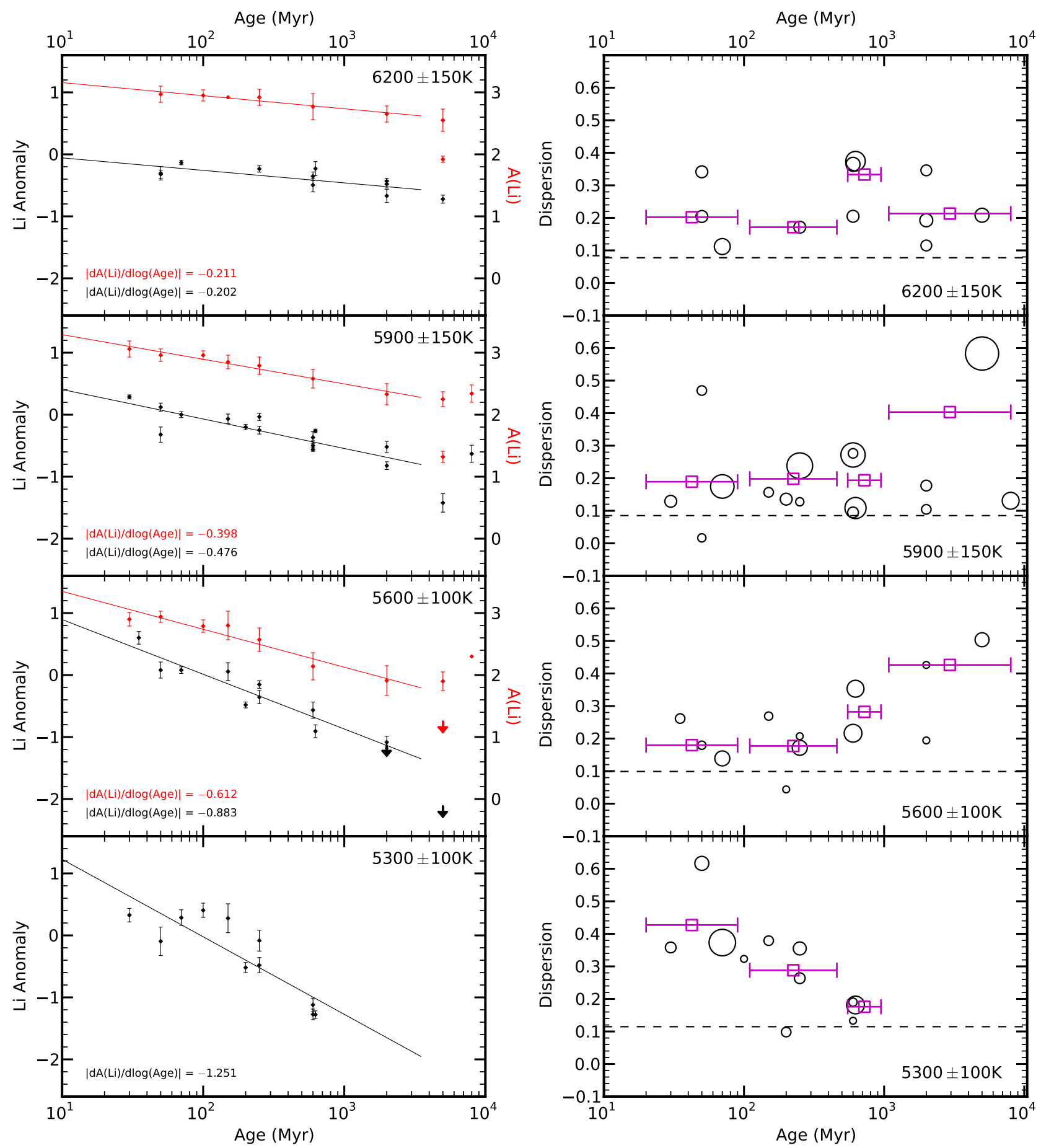

FIG. 12.- The timescales of Li depletion in anomaly space, and the development of Li dispersion at fixed mass. Left: Li data from SR05 (red points, right scale) compared to the same data detrended with the methods of $\S 4$ (black points). The red and black lines show the best fit power laws calculated up to $3 \mathrm{Gyr}$, with slopes reported in the lower left of each panel. The detrended clusters show a stronger mass trend than the absolute clusters. Right: The dispersion of each cluster considered in the left, with the sample size represented by the radius of the individual points. The dashed lines represents the absolute noise floor, and purple squares represent a weighted average of points within each age bin. Dispersion is found to emerge early in many clusters, and stay relatively constant for late F stars, rise marginally over time in solar analogs, and decrease up to $1 \mathrm{Gyr}$ in cool stars. The scatter of dispersions at fixed age suggest true cosmic variance in cluster Li patterns. 
inferred anomaly. This is somewhat mitigated by the correlation between $T_{\text {eff }}$ and $\mathrm{A}(\mathrm{Li})$ errors (see $\S 2.2$ ), but the effect may still be important. The second source is uncertainty in the metallicity of the cluster. If we detrend data with a SSM calculated for an improper $[\mathrm{Fe} / \mathrm{H}]$, the pre-MS mass trend will not be completely removed. This will leave behind a systematic offset within the $T_{\text {eff }}$ bin, which can introduce additional scatter. Finally, random errors in EW(Li) measurements will impart scatter. To assess the impact of these factors, we randomly generated 1000 stars within each $T_{\text {eff }}$ bin. The stars were assigned true $\mathrm{A}(\mathrm{Li}) \mathrm{s}$ based on their temperature, by interpolating in a SSM LDP calculated with solar metallicity. We assigned each an observed $T_{\text {eff }}$ error by randomly sampling a Gaussian with $\sigma=50 \mathrm{~K}$, and propagated this error into the observed $\mathrm{A}(\mathrm{Li})$. When this was done, we assigned each a $\sigma=10 \%$ Gaussian random EW(Li) error, and altered the inferred $\mathrm{A}(\mathrm{Li})$ according. We believe $10 \%$ to be a conservative estimate of the relative error of Li EWs within a single sample; although larger systematic errors between different studies may be present, this does not impact a dispersion measurement for a single data set. Finally, we detrended the stars against a SSM calculated with $[\mathrm{Fe} / \mathrm{H}]= \pm 0.1$ dex from the fiducial value. We performed this experiment 1000 times, and calculated the standard deviation within each bin. We found that in no case did the additional dispersion exceed 0.12 dex, implying that this method is reasonably stable to errors in photometry, spectroscopy, and composition. We represent this absolute noise floor by the dashed lines in each right-hand panel of Fig. 12; values above this noise floor can be considered detections of dispersion.

This figure demonstrates that dispersion is a generic feature of cluster LDPs at all ages. It is present in some large samples as early as 100-200 Myr, suggesting an early origin for the dispersion in some clusters. However, dispersion is undetected some very old clusters. This could be because of the small number of stars with known Li abundances in some clusters, or could imply that dispersion is not present in all systems. Clusters of equal age can show significantly different dispersions, and some clusters of dissimilar age show equivalent dispersions; this again could be due to the quality of the data sets, or signify cosmic variance. To examine the mean trends, we grouped the clusters into 4 age bins, calculated the mean dispersion within each, weighing data points by their sample size, and plotted the results as purple squares. The $6200 \mathrm{~K}$ bin shows that dispersion is, on average, present at all ages, but does not necessarily grow over time. Even the cluster with the greatest dispersion, M67, agrees with the mean trend in this $T_{\text {eff }}$ range. The $5900 \mathrm{~K}$ bin shows the most clusters are confined to a small band of dispersions, with only a few outliers. There is again little evidence of dispersion evolution in this range, although some outlying clusters are now apparent. The $5600 \mathrm{~K}$ bin shows a more convincing rising trend, with the five largest samples rising over time; this is evidence that in this temperature range, the spread can increase along the MS. Finally, in the coolest bin we see a trend of decreasing dispersion with age. This is related to the large dispersion at fixed temperature seen in young systems, such as the Pleiades, which is suppressed in some intermediate-age systems, such as the Hyades. This implies that the most abundant stars at ZAMS (i.e. the fastest rotating) deplete $\mathrm{Li}$ more rapidly on the MS, such that the dispersion decreases over the first Gyr of MS evolution.

These plots reveal great complexity in the development of Li dispersion in open clusters, and raise several questions. First, can cluster Li dispersions really differ at the same age? The most uninteresting explanation for this figure would be that no underlying difference exists, and that the data sets we employ are sampling the same distribution, but with poor enough statistics that noise is dominating. We can clearly rule this out in some instances, such as the comparison between M67 and NGC 188. The younger M67 has both a more depleted upper envelope, and a substantially larger scatter than NGC 188. There are 13 stars in our NGC 188 sample between $5700 \mathrm{~K}$ and $6000 \mathrm{~K}$, all of which are more abundant than $\mathrm{A}(\mathrm{Li})=1.8$. There are 29 stars in the M67 sample in this range, 13 of which are more abundant than 1.8, and 16 of which are less abundant than this value. It seems highly unlikely that these fractions could be drawn from the same distribution. Furthermore, the total range of abundances is $\sim 0.5$ dex for NGC 188 , and $\gtrsim 1.7$ dex for M67, again highly inconsistent with being drawn from the same distribution. This strongly implies that the depletion histories of these two clusters, and therefore clusters in general, can differ.

Second, why does the magnitude of dispersion vary from cluster to cluster? The source of this variance may be related to the initial conditions of open clusters. The early onset of dispersion described above is qualitatively consistent with the picture expected from rotational-mixing. In short, the greatest spread in rotation rates occurs in the first $200 \mathrm{Myr}$ of the MS, so the largest degree of differential depletion would occur then. If the initial AM distribution can vary between clusters, then the Li dispersion that ultimately develops will vary as well. Open cluster rotation distributions typically show a narrow, densely populated band of converged rotators, and a sparsely populated tail towards more rapid rotation (e.g. M37 - Hartman et al. 2009; Pleiades - Hartman et al. 2010; Irwin \& Bouvier 2009, and refs. therein). In this paradigm, the tail would produce the most Li-depleted objects, and the converged stars should show smaller, nearly uniform depletion factors. If rotation is truly responsible for the cosmic variance in LDPs, then the fractional size of the rapid-rotator tail must be what differentiates high-dispersion clusters from low-dispersion clusters. However, given the small fraction of stars in the high-velocity tail in some clusters (e.g. Hartman et al. 2010, Fig. 14), large Li data sets may be needed for a robust measure of the dispersion. Another cluster initial condition that could contribute to the scatter is the number density of members. Stellar mergers are more likely in dense environments, and low mass stars that undergo normal depletion on the pre-MS, then merge into a higher mass star during the MS, would appear scattered below the mean trend in a present day LDP. While both of these scenarios are qualitatively sensible, detailed work must be done to establish their predictions for the range of plausible resulting $\mathrm{Li}$ patterns.

Finally, does the dispersion of Li correlate with other observables? Another light element that can be destroyed by mixing on the MS is beryllium. One would expect a corresponding spread of this element, which 
burns at a temperature of 3.5 million $K$, to be present in clusters with a large Li dispersion. Since Be survives to deeper layers in stars, the expected pattern for a given theoretical scenario will differ between the elements, but should correlate. Therefore, dual data sets would place additional constraints on theoretical models seeking to explain MS dispersion. Additionally, rich rotation data sets in clusters with extensive Li measurements have recently been obtained (M34 - Meibom et al. 2011; Pleiades - Hartman et al. 2010). If the early AM distributions of clusters impact their Li evolution, this may be observable in the present day rotation patterns of clusters with differing LDPs.

\section{DISCUSSION}

\subsection{Does Metallicity Impact Pre-MS Lithium Depletion?}

In SSMs, the stronger opacity resulting from an increased metal abundance suppresses the efficiency of radiative energy transport. The result is an increased $T$ gradient, which causes the depth of the surface CZ to increase. Consequently, $T_{B C Z}$ increases, and the rate of Li destruction goes up. This causes pre-MS Li depletion in stars to be highly sensitive to metallicity. We have relied on the validity of this strong theoretical prediction in our calculation of the lithium anomaly. However, the existence of this metallicity effect has been questioned by some authors from the observational side. Jeffries \& James (1999) argued that the Li distribution of the open cluster Blanco 1 is identical to that of the similarly aged Pleiades, despite being claimed to be 0.1-0.2 dex richer in metals. Sestito et al. (2003) reached a similar conclusion from a comparison of NGC 6475 and M 34. While puzzling at the time, these results have since been called into question by more recent calculations of the iron abundance of Blanco $1(+0.04 \pm 0.02$; Ford et al. 2005), NGC $6475(+0.03 \pm 0.02$; Villanova et al. 2009), and M $34(+0.07 \pm 0.04$; Schuler et al. 2003). Nevertheless, there remain open clusters of differing $[\mathrm{Fe} / \mathrm{H}]$ with apparently similar empirical LDPs, particularly the trio of $\sim 1.5$ Gyr old clusters: NGC 752 , NGC 3680, and IC 4651 (Sestito et al. 2004; AnthonyTwarog et al. 2009, AT09 hereafter). Differential depletion imprinted at ZAMS should persist as cluster evolve on the MS, so these authors argue that the similarity of the current Li patterns suggests pre-MS depletion cannot depend on $[\mathrm{Fe} / \mathrm{H}]$. Can this result be reconciled with standard stellar theory? In this section, we address this question by comparing these cluster patterns in absolute and anomaly space. We apply this same analysis to the Hyades and NGC 6633, two 600 Myr old clusters with substantial composition differences. If metallicity is an important factor in determining ZAMS abundance, the metal poor cluster patterns should lie above the metal rich patterns in absolute space, and on top of them in anomaly space.

We first examine the LDPs of the three clusters mentioned above: NGC 752, NGC 3680, and IC 4651. Iron abundances and ages for each are reported by AT09: $[\mathrm{Fe} / \mathrm{H}]=-0.05$ and age $=1.45 \mathrm{Gyr}$ for NGC $752,[\mathrm{Fe} / \mathrm{H}]$ $=-0.08$ and age $=1.75$ Gyr for NGC 3680, and $[\mathrm{Fe} / \mathrm{H}]=$ +0.13 and age $=1.5$ Gyr for IC 4651. These abundances are due to high resolution spectroscopy, and appear robust in their relative values (see the discussion in AT09 and references therein). We draw BV photometry and Li EWs from Sestito et al. (2004) for NGC 752, from AT09 for NGC 3680, and from Randich et al. (2000) for IC 4651. We synthesize this data into effective temperatures and $\mathrm{A}(\mathrm{Li}) \mathrm{s}$ using the method described in $\S 2.2$, and compute SSM Li predictions using the methodology of $\S 2.3$.

These data are presented in the left panel of Fig. 13. NGC 3680 (filled black) and NGC 752 (empty black) have similar $\mathrm{Fe}$ abundances, and unsurprisingly show similar median Li trends. IC 4651 (empty red) is $\sim 0.2$ dex richer in Fe, but lines up well in absolute space with the other clusters. At face value this appears surprising, given the assumption of a strong $[\mathrm{Fe} / \mathrm{H}]$ dependence of ZAMS Li patterns. However, since IC 4651 data only exists for stars with $T_{\text {eff }} \gtrsim 5800 \mathrm{~K}$, a regime that is particularly insensitive to composition $(\Delta[\mathrm{Fe} / \mathrm{H}]=0.2 \mathrm{dex}$ $\rightarrow \Delta \mathrm{A}(\mathrm{Li})=0.3$ dex at $5800 \mathrm{~K} ; \S 3.1)$, modest scatter may obscure the relative depletion signal. The bottom left shows the results of Kolmogorov-Smirnov (K-S) tests, which demonstrates that in each case, the clusters cannot be statistically distinguished. This supports the visual impression that the cluster LDPs are similar.

The right hand side shows the same data detrended with respect to each clusters' SSM prediction, as described in $\S 4$. In the anomaly plane, the relative cluster distributions remain similar. NGC 3680 and NGC 752 have shifted very little with respect to one another, but IC 4651 has trended upward relative to the other clusters. The locus of IC 4651 data appears to lie near the top of the distribution of NGC 3680 and NGC 752, but this visual impression is largely due to a single data point at the top right of the IC 4651 distribution. These clusters still cannot be distinguished by a K-S test, and show no statistical improvement over the comparison in absolute space. This suggests that the cluster LDPs appear similar because they are in a temperature range that is particularly insensitive to metallicity. Furthermore, we cannot rule out differences in the initial cluster abundance of a few tenths of a dex, which could bring these clusters into superior agreement. Cummings (2011) finds a higher initial abundance for more iron rich clusters, so IC 4651 may have begun life at a slightly higher abundance, and suffered slightly higher depletion to wind up at a similar location in anomaly space at 1.5 Gyr. Given the weak dependence of the models on metallicity in this regime, the similarity of the LDPs in anomaly space, and the remaining uncertainties in initial $\mathrm{Li}$ abundance and relative $[\mathrm{Fe} / \mathrm{H}]$, we conclude that these clusters do not convincingly demonstrate that composition is unimportant in determining the ZAMS Li pattern.

Another pair of clusters that are similar in age, and whose relative metallicities have been well-determined, are the Hyades and NGC 6633. The latter is a $\sim 600 \mathrm{Myr}$ old (Strobel 1991) open cluster with an iron abundance that is $0.206 \pm 0.40$ dex less than the Hyades (Jeffries et al. 2002, J02 hereafter). This is a secure relative value, since both used high resolution spectroscopy, and the analysis of NGC 6633 was carried out in precisely the same way as the analysis that derived the benchmark Hyades data. A substantial Li data set is available for this cluster, due as well to J02. With our prior Hyades $[\mathrm{Fe} / \mathrm{H}]$ of +0.135 , we derive $[\mathrm{Fe} / \mathrm{H}]=-0.071 \pm 0.040$ for NGC 6633. We then compute SSM Li predictions for 


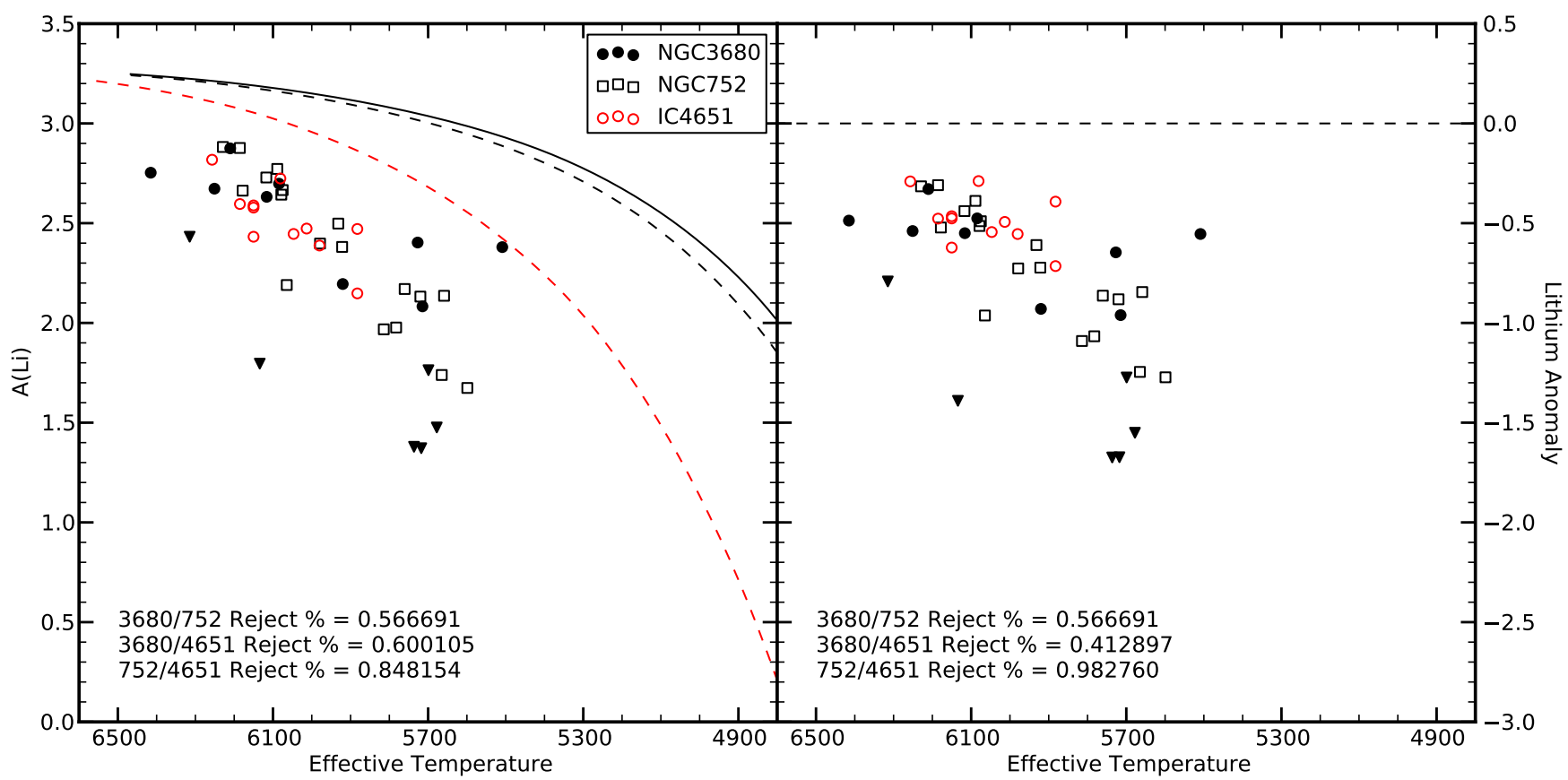

FIG. 13. - A comparison of three $2 \mathrm{Gyr}$ old clusters in $\mathrm{A}(\mathrm{Li})$ and $\mathrm{Li}$ anomaly space, which demonstrates that they do not rule out metallicity effects on the pre-MS. NGC 3680 (filled black circles), NGC 752 (empty squares), and IC 4651 (empty red circles) data and shown alongside their SSM predictions (solid black, dashed black, and dashed red lines, respectively) in the left panel, and subtracted from their SSM predictions in the right. IC 4651 is $\sim 0.2$ dex richer in metals, but lines up well with the other clusters in both absolute and anomaly space. The inconclusive rejection probabilities shown in the bottom left of each panel imply that in neither case can the abundance distributions be statistically distinguished.

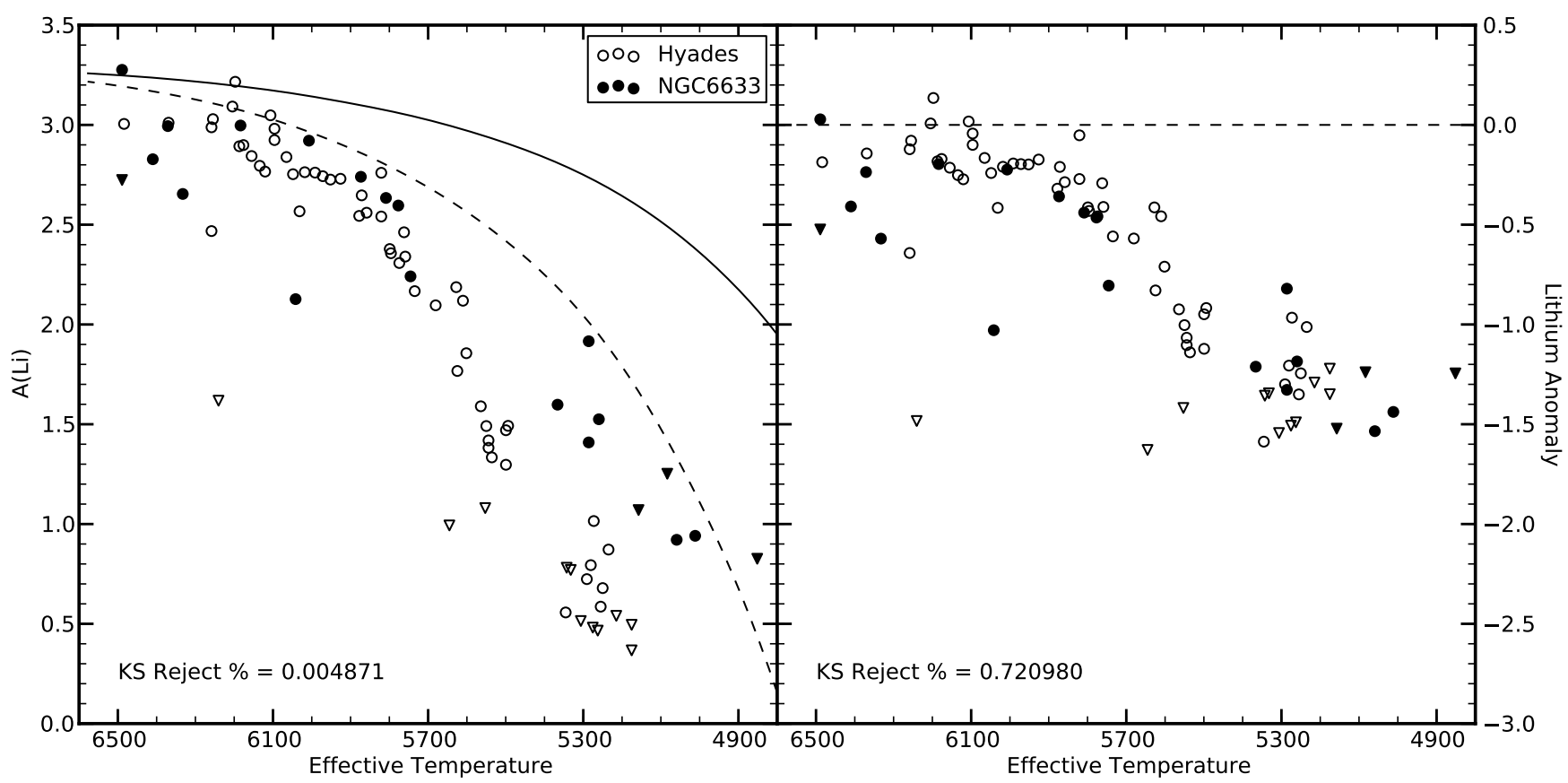

FIG. 14.- A comparison of two $600 \mathrm{Myr}$ old clusters in $\mathrm{A}(\mathrm{Li})$ and anomaly space, which demonstrates that metallicity likely impacted their pre-MS abundance distributions. Hyades (empty) and NGC 6633 (filled) data are shown alongside their SSM predictions (dashed black and solid black lines, respectively) in the left, and subtracted from their SSM predictions in the right. The null hypothesis that the $\mathrm{Li}$ distributions are equal can be rejected at high confidence in $\mathrm{A}(\mathrm{Li})$ space, but cannot be rejected in detrended space. This suggests that SSMs accurately predict the relative depletion of these clusters. 


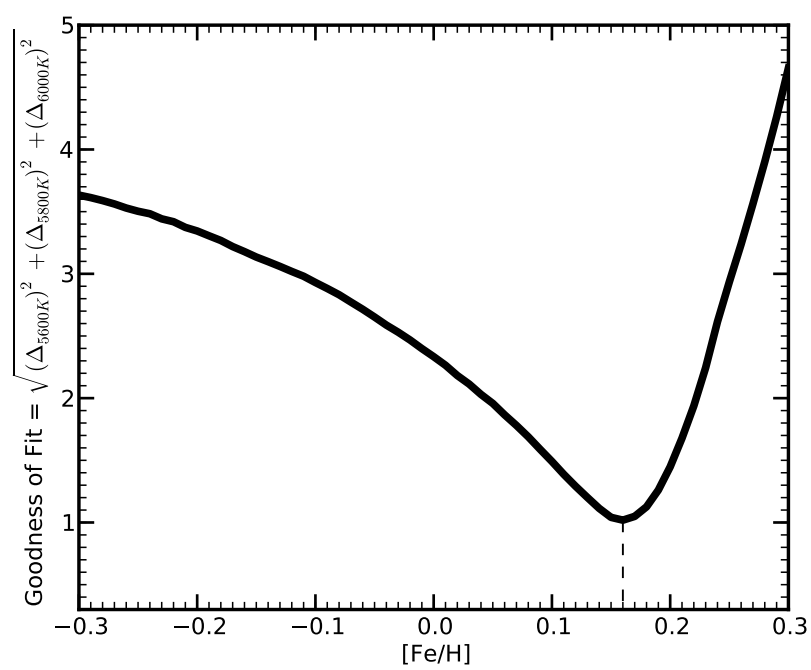

FIG. 15. - The quality of agreement between detrended Hyades $\mathrm{Li}$ data and detrended Praesepe Li data, versus the metallicity assumed when detrending Praesepe. The best fit $[\mathrm{Fe} / \mathrm{H}]$ is at +0.16 , implying that this is the most likely metallicity for the Praesepe, according to the method described in $\S 6.1$. This is in good agreement with the most recent high-resolution spectroscopic measurement of $+0.12 \pm 0.04$ (Boesgaard et al. 2013).

this cluster, draw BV photometry and Li EWs from J02, and synthesize the data into effective temperatures and abundances using the procedure described in $\S 2$.

The results are presented in Fig. 14. The left panel shows the Hyades (empty circles) and NGC 6633 (filled circles) abundances compared with one another. The $\mathrm{Li}$ distributions are similar above $5700 \mathrm{~K}$, just as in the above case. However, below 5300K the median NGC 6633 trend is clearly more abundant than the Hyades stars, and a K-S test shows that the probability that these data sets are equivalent is $<0.005$. This discrepancy cannot be due to age differences, since if the Hyades and NGC 6633 had identical Li patterns at ZAMS, the Hyades would need to be nearly twice the age of NGC 6633 to produce the difference between median abundances at $5300 \mathrm{~K}$. This would imply either NGC 6633 is 300 Myr old or the Hyades is 1200 Myr old, both of which are strongly inconsistent with their measured ages. Next, we detrend the clusters and plot them in the right panel of Fig. 14. The agreement between the clusters is significantly improved when viewed in anomaly space, and both the warm and cool NGC 6633 stars appear to lie on top of the Hyades stars. A K-S test reveals that the populations can no longer be statistically distinguished ( $\sim 0.72$ rejection probability), suggesting that the magnitude of differential depletion between these two clusters is accurately predicted by SSMs. This strongly implicates metallicity as the cause of the difference between the Hyades and NGC 6633 LDPs.

The similarity of these two clusters in detrended space, and the paucity of secure counter-examples, leads us to conclude that metallicity does impact pre-MS Li destruction. This implies that our calculation of the lithium anomaly is a valid measure of MS depletion. To solidify this result, and to quantify the magnitude of the preMS metallicity effect, the LDPs of additional groups of similar-aged clusters must be compared. In particular, their relative compositions must be robustly determined. Recent work by Heiter et al. (2013) demonstrates that differences in the analysis method are a larger source of error in $[\mathrm{Fe} / \mathrm{H}]$ determinations than differences in the quality of observations when using high-resolution spectra. This suggests that a uniform re-analysis of highresolution $\mathrm{Fe}$ observations may be able to resolve this issue. Finally, we have shown that LDP similarity is difficult to establish with solar analogs, so future work should focus on obtaining large samples of Li measurements of cool stars. The temperature range is critical, since SSMs predict that a 0.2 dex difference in iron abundance leads to a 0.2 dex difference in $\mathrm{Li}$ at $6000 \mathrm{~K}$, but a 1.2 dex difference at $5000 \mathrm{~K}$. The former may not be detectable, but the latter certainly is.

The extreme sensitivity of Li depletion to metallicity opens the exciting possibility of using Li to infer cluster composition. If the detrended LDPs of equal age clusters are morphologically similar, then the composition of a cluster can be constrained by finding the SSM LDP that produces the best agreement with a cluster of known composition in anomaly space. We tested this concept by estimating the metallicity of the $\sim 700$ Myr old Praesepe (Salaris et al. 2004) using the well-constrained, and similar aged, Hyades. The $[\mathrm{Fe} / \mathrm{H}]$ of Praesepe has been a controversial subject: it has been measured as low as $[\mathrm{Fe} / \mathrm{H}]=+0.04 \pm 0.04$ (Boesgaard \& Friel 1990), and as high as $[\mathrm{Fe} / \mathrm{H}]=+0.27 \pm 0.10$ (Pace et al. 2008), with the most recent, high-resolution spectroscopic study finding $[\mathrm{Fe} / \mathrm{H}]=+0.12 \pm 0.04$ (Boesgaard et al. 2013). We first analyzed Praesepe Li EW and BV data from Soderblom et al. (1993b) using the methods described in $\S 2.2$, with the metallicity of Boesgaard et al. (2013). Using the machinery of $\S 2.3$, we created 0.7 Gyr SSM predictions for $[\mathrm{Fe} / \mathrm{H}] \mathrm{s}$ ranging from -0.30 to 0.30 , in steps of 0.01 , and detrended the Praesepe data with each. We then compared each detrended pattern with the Hyades anomaly pattern, shown in Fig. 14. To establish a quantitative measure of agreement, we calculated the difference in the median anomalies of the two clusters in three $T_{\text {eff }}$ bins: $5500-5700 \mathrm{~K}, 5700-5900 \mathrm{~K}$, and 5900-6100K. We then weighted each point by the MAD of the data around the median, and added the differences in quadrature. The resulting $[\mathrm{Fe} / \mathrm{H}]$ vs. goodness-of-fit curve is shown in Fig. 15, with lower goodness-of-fit values demonstrating superior agreement. As can be seen, our best fit $[\mathrm{Fe} / \mathrm{H}]=+0.16$, consistent at $1 \sigma$ with the most recent high-resolution spectroscopy estimate for Praesepe.

We note a few caveats to this method. First, there is a partial degeneracy between cluster $[\mathrm{Fe} / \mathrm{H}]$ and the initial $\mathrm{Li}$ abundance of its members. We assessed the impact of this effect by changing the initial $\mathrm{Li}$ abundance of our Praesepe models, and recalculating the best fit $[\mathrm{Fe} / \mathrm{H}]$. We found that the best fit $[\mathrm{Fe} / \mathrm{H}]$ changed to +0.10 when the initial Praesepe abundance was decreased by 0.1 dex, and +0.20 when the initial Praesepe abundance was increased by 0.1 dex. We expect that this is a minor effect, since clusters must be coeval for this method to work. Furthermore, an uncertainty of \pm 0.05 dex in $[\mathrm{Fe} / \mathrm{H}]$, from an uncertainty of \pm 0.1 dex in initial Li abundance, is small compared to quoted errors on most spectroscopic metallicities. Second, we did not re-derive the stellar parameters of Praesepe members for each $[\mathrm{Fe} / \mathrm{H}]$. The $T_{\text {eff }}$ scale is somewhat sensitive to $[\mathrm{Fe} / \mathrm{H}]$, so a more careful calculation should take this effect into account when determining goodness of fit. Nevertheless, this proof-of- 
concept demonstrates that this method merits further attention.

In summary, we have shown that the metal abundance of stars is a crucial factor in shaping pre-MS Li abundance patterns. A trio of 2 Gyr old clusters with different compositions but similar LDPs were shown to be consistent with the prediction of additional depletion in metal-rich stars. Since the stars in these samples are all in an $T_{\text {eff }}$ regime where the metallicity effect is weak, their apparent similarity in $\mathrm{A}(\mathrm{Li})$ space does not constitute evidence against the standard picture. On the other hand, two $600 \mathrm{Myr}$ old clusters of differing composition were shown to have significantly different average $\mathrm{Li}$ abundances in absolute space. Once detrended into anomaly space, the clusters became statistically indistinguishable, demonstrating that SSMs accurately predicted the difference between their patterns. Finally, we have shown that the strong dependence of LDPs on composition can be used to constrain the metallicity of open clusters, by comparing them to an equal-age clusters with well known metallicity.

\subsection{Suppressed Convection and the Radius Anomaly as the Origin of the Lithium Dispersion in Young Cool Dwarfs?}

An intriguing feature of the Pleiades Li pattern that standard stellar theory cannot explain is the large abundance dispersion in stars cooler than $5500 \mathrm{~K}$. While SSMs predict that $\mathrm{Li}$ content is uniquely determined by age, mass, and composition, a spread of $~ 1.5$ dex in $\mathrm{A}(\mathrm{Li})$ was discovered in the cool Pleiads by Duncan \& Jones (1983), and later confirmed by S93. The latter authors further showed that in this temperature regime, fast rotating stars are on average less depleted than their slow rotating counterparts (S93, Fig. 2). This is counter-intuitive, because rapid rotation is expected to, if anything, drive additional mixing, and therefore deplete more Li (Pinsonneault et al. 1989).

In the years since this discovery, several authors have attempted to explain this dispersion through surface effects that impact $\lambda 6708 \mathrm{Li}$ I EWs. One possibility is chromospheric activity affecting the physics of line formation (S93; Carlsson et al. 1994; Stuik et al. 1997; Jeffries 1999). These authors argued that such an effect would impact other lines as well, such as the $\lambda 7699$ K I resonance line. S93 initially found a commensurate spread in the $\lambda 7699 \mathrm{~K} \mathrm{I}$ and $\lambda 6707 \mathrm{Li} \mathrm{I}$ features in cool Pleiads, supporting the notion that the Li spread is spurious. However, high resolution spectra obtained by King et al. (2010; JK10 hereafter) found no such scatter in the $\mathrm{K} I$ line, while confirming a spread of $\gtrsim$ a factor of 2 in the $\mathrm{Li}$ I line. Another potential effect is rotational broadening of the Li absorption line in rapid rotators (Margheim et al. 2002), which could naturally explain why fast spinning stars appear more abundant. However, Margheim (2007) later demonstrated that this cannot account for the full dispersion, since abundances derived though EW analysis and abundances derived through rotationallybroadened spectral synthesis agreed for the majority of the stars. Finally, if cool Pleiads have a large spot filling factor, the neutral Li I line could be enhanced, as the ionization fraction would be lower on large swaths of the surface. However, JK10 demonstrated that this cannot account for the full effect, as the maximum fill- ing factors necessary to develop the observed EW spread would result in a $\mathrm{V}$-band magnitude dispersion of $\sim 1$ mag, a feature not observed in Pleiades HR diagrams. It appears that a combination of these effects may contribute to the total dispersion, but that a true underlying Li spread must exist (JK10).

Several mechanisms have been proposed as the cause of this effect. These include episodic accretion during the proto-stellar phase altering the interior temperature (Baraffe \& Chabrier 2010), star-disk coupling inducing strong internal mixing shears (Eggenberger et al. 2012), and increased stellar radii impacting depletion efficiency (K10). In this section, we investigate a version of this final possibility. The rate of pre-MS Li depletion in a star is exquisitely sensitive to $T_{c e n}$ during the proto-stellar epoch $(\S 1)$. If some mechanism reduced $T_{c e n}$ below its standard predictions during this time period, the Li depletion rate would be severely suppressed, and the ZAMS abundance would be higher than anticipated. As described in the introduction, numerous studies have identified pre-MS and MS stars whose radii are inflated $\approx$ $5-15 \%$ relative to standard theory. The presence of this radius anomaly would reduce the pressure in the central regions, thus decreasing the temperature required to maintain equilibrium, and slowing the rate of $\mathrm{Li}$ burning.

In $\S 6.2 .1$, we briefly discuss potential underlying causes of the radius anomaly, and describe how such anomalies could induce the observed Li pattern. In $\S 6.2 .2$, we present inflated stellar models of the Pleiades, and conclude that radius anomalies of the observed magnitude can suppress Li depletion by the required amount. In $\S 6.2 .3$, we extend this analysis to six additional young clusters, and show that radius anomalies can explain the general pattern of LDP evolution on the pre-MS. In $\S 6.2 .4$, we discuss implications of radius anomalies for pre-MS evolutionary tracks, stellar initial mass function (IMF) measurements, and the ages of young open clusters. Finally, in $\S 6.2 .5$ we summarize the results of this section and suggest directions for future study.

\subsubsection{The Radius Anomaly}

Radius anomalies have been observed in a large number of systems (see $\S 1$ ). While the underlying cause is not yet understood, several explanations have been put forward. Accretion from a circumstellar disk may impact the proto-stellar radius by adding thermal energy to the envelope (Palla \& Stahler 1992), but the existence of radius anomalies at a few $100 \mathrm{Myr}$ (e.g. YY Gem; Torres \& Ribas 2002), long after the T Tauri phase, makes this unlikely to be the sole culprit. Theoretical expectations for a given system can also be greatly impacted by errors in metallicity, as stellar radii are sensitive to opacity. This is particularly apparent in interferometric measurements of $\mathrm{M}$ dwarfs (Berger et al. 2006), and may indicate missing opacity sources in very cool stars. Nevertheless, radius anomalies persist in many systems with very well measured composition (e.g. CM Dra; Terrien et al. 2012), so this cannot fully explain the phenomenon.

The current leading explanation is the presence of magnetic fields in low-mass stars (Mullan \& MacDonald 2001; Chabrier et al. 2007; Morales et al. 2008; MacDonald \& Mullan 2012; Feiden \& Chaboyer 2013), which can impact the radius in two ways. First, strong magnetic activity can increase the coverage of spots on the stel- 


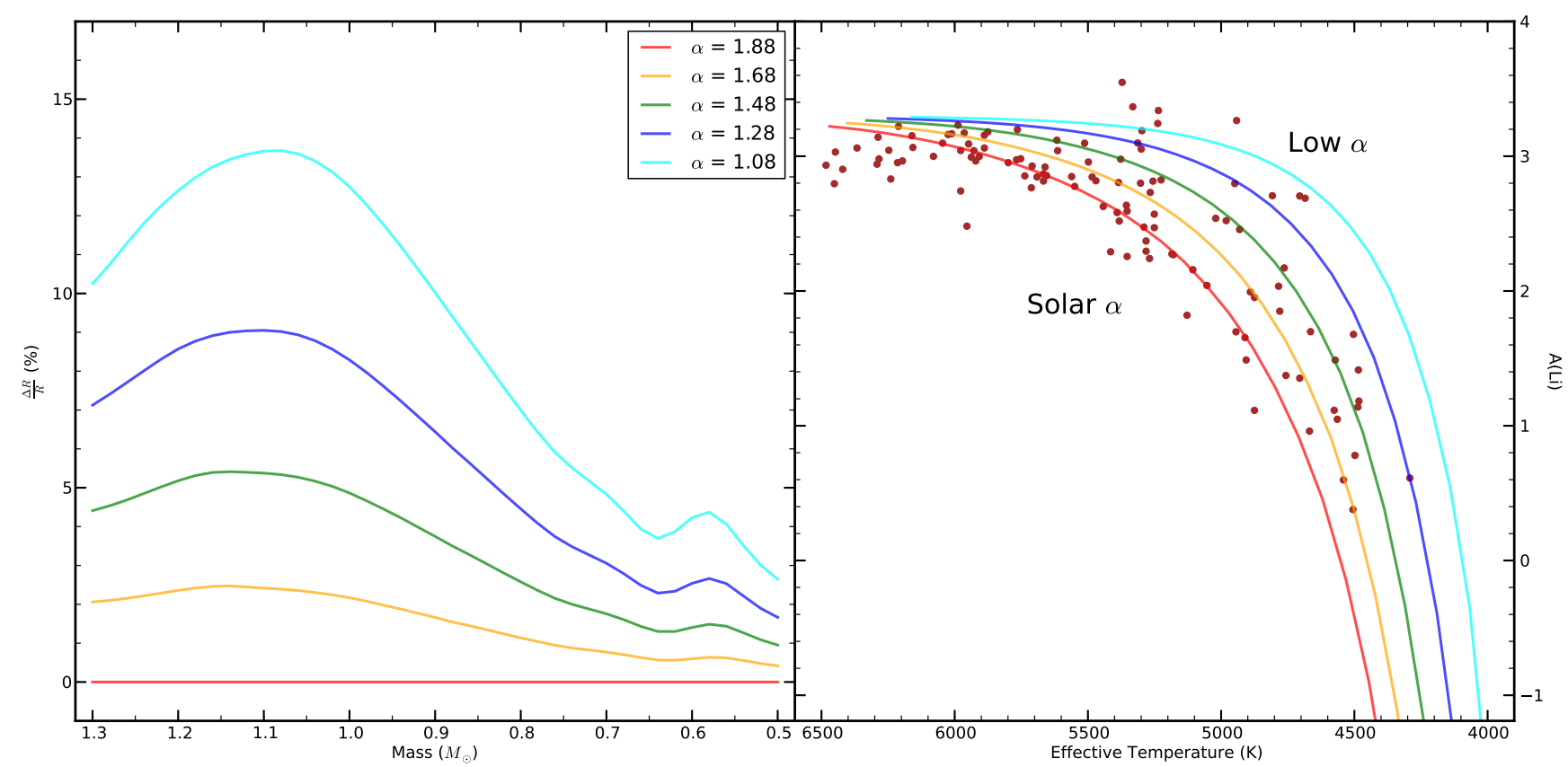

FIG. 16. - The radius anomalies and Li abundances resulting from inflation on the pre-MS. The left panel shows the fractional change in the radius of stellar models with reduced mixing-lengths, relative to the solar-calibrated mixing-length model (red line), as a function of mass. Radius anomalies of $\sim 5-15 \%$ on the pre-MS can inhibit SSM depletion enough to account for most cool stars in the Pleiades, as seen in the right panel. This paradigm predicts a large spread developing at fixed effective temperature around 10 Myr.

lar surface, which reduces $T_{\text {eff }}$ and puffs out the envelope (Andronov \& Pinsonneault 2004). Second, it can inhibit the efficiency of thermal convection, which creates a stronger radiative energy gradient to compensate for the reduced convective energy flux. This enhances the temperature gradient, which leads to a lower surface temperature and causes the radius to expand. This theory is supported by the discovery of a correlation between the radius anomaly and the strength of surface magnetic field proxies. Stars with stronger coronal activity, often inferred as the ratio of X-ray (or $\mathrm{H} \alpha$ ) to bolometric luminosity, show a larger fractional disagreement between their measured radii and SSM predictions (López-Morales 2007; Clausen et al. 2009; Stassun et al. 2012). Coronal activity also correlates with rotation on the MS (e.g. Wilson 1966; Kraft 1967; Fleming et al. 1989; Bouvier 1990), implying that stars with the most inflated radii may also the most rapidly rotating. Young clusters, such as the Orion Nebula Cluster, possess a large range of X-ray luminosities (Preibisch et al. 2005) and rotation rates (Stassun et al. 1999; Herbst et al. 2001; Herbst et al. 2002) at fixed mass, suggesting that a range of radius anomalies, as large as $0-15 \%$, may be present in young clusters.

This presents a plausible explanation for the Li spread in the Pleiades: the most rapidly rotating stars are puffed up relative to standard models on the pre-MS, causing their central temperatures to decrease. This greatly inhibits Li depletion during the pre-MS burning phase, and so they lie significantly above the SSM LDP at the age of the Pleiades. The slowest rotating stars have no radius anomaly, burn $\mathrm{Li}$ at a rate consistent with standard predictions, and therefore lie close to the SSM LDP. The result is a range of abundances between the standard prediction for a given $T_{\text {eff }}$, and the $\mathrm{Li}$ abundance produced by a star with the maximal radius anomaly. Moreover, the most inflated stars are also the most rapidly rotating, creating an observable correlation between $\mathrm{Li}$ abundance and surface rotation rate at $120 \mathrm{Myr}$. This is an elegant solution to this problem, as an empirically observed radius effect may simultaneously explain the abundance spread, why the median lies above the standard prediction, and why fast rotating stars are less depleted.

\subsubsection{Lithium and Radius in the Pleiades}

Before this possibility can be considered further, we must first show that radius anomalies of the observed magnitude can suppress $\mathrm{Li}$ burning by the required amount? To do this, we calculate standard stellar models with inflated radii. This is achieved by decreasing the mixing-length $(\alpha)$ in our calculations, which inhibits the efficiency of convection and puffs up the stellar envelope, similar to the effect of strong magnetic fields (Chabrier et al. 2007). We run the models to 120 Myrs, and display them in Fig. 16. The left panel quantifies the radius anomaly induced by each choice of $\alpha$, as a function of mass. The red line represents the solar-calibrated SSM ( $\frac{\Delta R}{R}=0$, by definition), and the yellow, green, blue, and cyan lines represent calculations where the mixinglength is progressively lower in steps of 0.2 . The largest anomalies for these models are $\sim 10-15 \%$ above $0.9 M_{\odot}$, and $\sim 5 \%$ for lower masses, consistent with the range of observed anomalies (Stassun et al. 2012).

The right panel of Fig. 16 shows the resulting LDPs, plotted alongside the Pleiades data from Fig. 1. These models were calculated with $[\mathrm{Fe} / \mathrm{H}]=+0.03$ (Table 1 ), and have been corrected for theoretical systematics using the method described in $\S 4$. In the absence of additional mixing mechanisms, the qualitative features of the Pleiades LDP are well reproduced by inflated models. The solar mixing-length LDP neatly traces the lower envelope of the data below $\sim 6000 \mathrm{~K}$, and the most inflated 


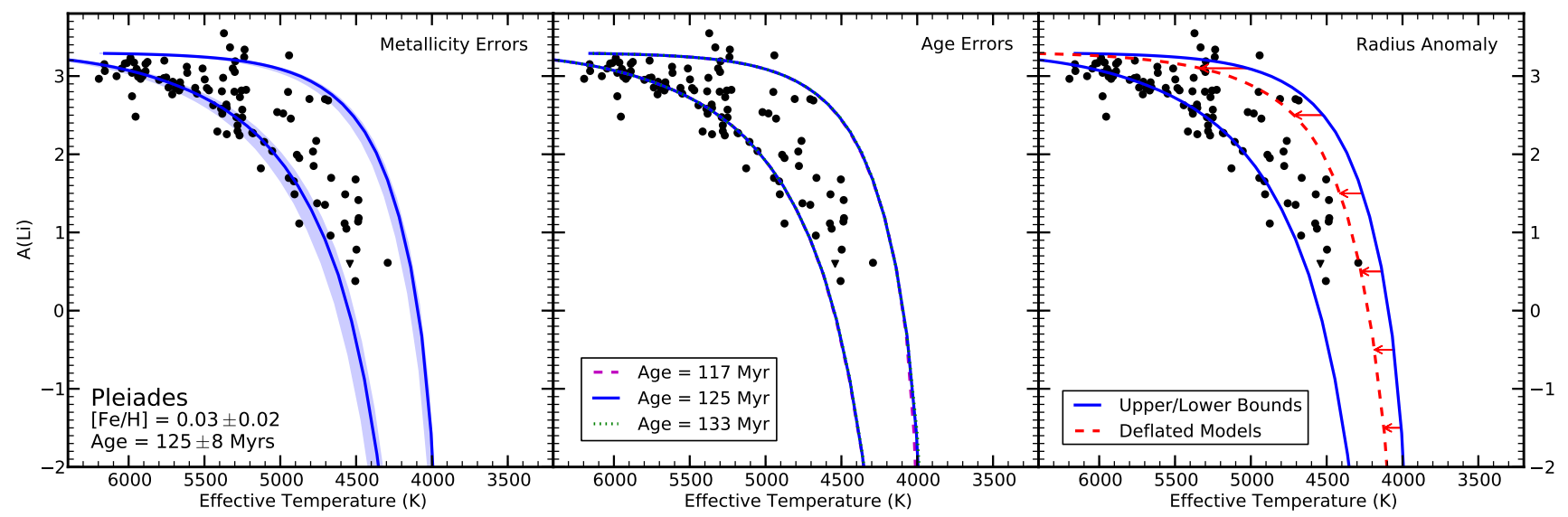

FIG. 17.- The effects of three sources of error on the agreement between the Li predictions of inflated stellar models and the empirical Pleiades LDP. Left: Blue lines represent inflated (upper) and standard (lower) models calculated with the best-guess cluster parameters for the Pleiades. The blue shaded bands represent the locations these models could lie given $1 \sigma[\mathrm{Fe} / \mathrm{H}]$ errors. Center: Blue lines again represent best-guess Pleiades models. The red dashed and green dotted lines represent the locations of the models given $1 \sigma$ age errors. Right: Blue lines represent the best guess models for the Pleiades, and the red dashed line represents the location of the upper envelope, once the models have deflated to their standard radius predictions.

LDP closely brackets the upper envelope of the distribution. Similar to the empirical data, the dispersion in the models is tight above 1 solar mass, and widens considerably towards cooler stars: $\Delta \mathrm{A}(\mathrm{Li})=0.21$ dex at $6000 \mathrm{~K}$, 0.52 dex at $5500 \mathrm{~K}, 1.18 \mathrm{dex}$ at $5000 \mathrm{~K}$, and $2.83 \mathrm{dex}$ at $4500 \mathrm{~K}$. Given errors in $T_{\text {eff }}$ and $\mathrm{A}(\mathrm{Li})$, the upper envelope of the distribution appears somewhere between the models with $\alpha=1.28$ and 1.08. This corresponds to radius anomalies $\sim 2-4 \%$ at $0.6 M_{\odot}, 4-7 \%$ at $0.8 M_{\odot}$, and $8-12 \%$ at 1.0 and $1.2 M_{\odot}$, well within the observed range of anomalies in rapidly rotating systems.

While this qualitative agreement is excellent, there are a few additional factor to account for. First, the correlation between rotation rate and radius anomaly suggests that stars converge to their standard model radii as they spin down on the MS. When the initially inflated models deflate to their solar-calibrated radii, the surface temperature will increase, and the stars near the top of the $\mathrm{Li}$ distribution will move towards the left in our plots. This may reduce the apparent $\mathrm{Li}$ dispersion in Fig. 16. Second, errors in the age and metallicity of the Pleiades will alter the location of the theoretical LDPs. To address these issues, we recast our models in Fig. 17. The lower blue line in each panel reflects the standard model LDP at the fiducial metallicity and age, and the upper blue line reflects inflated models calculated with the mixinglength $\alpha=\alpha_{\odot}-0.8$. The shaded blue regions in the left panel represent the range of locations these bounds could lie in, given the quoted $1 \sigma[\mathrm{Fe} / \mathrm{H}]$ errors. The predicted bounds still neatly bracket the available data, confirming the qualitative suggestion of Fig. 16. The central panel shows two additional upper and lower LDPs, calculated for $1 \sigma$ age errors. There is no substantial difference between the theoretical prediction at these three ages, suggesting that the relevant Pleiades stars have entered the MS, and no longer change on Myr timescales. Finally, the right panel of Fig. 17 shows an LDP that was inflated on the pre-MS, but whose radii have converged to standard predictions (red line). SSM Li depletion is completed before MS spin-down commences in all stars, so the $\mathrm{Li}$ abundance is not affected when stars deflate. The LDP therefore shifts only in $T_{\text {eff }}$, and not in $\mathrm{A}(\mathrm{Li})$, as represented by the red arrows. The deflated models still trace the upper envelope of the Pleiades well, confirming that the cool star Li dispersion persists after substantial MS spin-down has occurred.

With these effects included, our models selfconsistently explain many features of the Pleiades LDP: 1) the small dispersion in hot stars, 2) the median abundance of hot stars, 3) the dispersion in cool stars, 4) the locations of the upper and lower envelopes of the cool star distribution, and 5) the rotation-Li correlation seen below $5500 \mathrm{~K}$. Given that these models were calibrated only to reproduce the empirically-observed radius anomaly, without any regard for the Li predictions, the excellent agreement between data and theory strongly suggests that pre-MS inflation is the cause for the Pleiades Li dispersion.

\subsubsection{Additional Clusters}

As a test of the generality of this picture, we extend this full analysis to six additional young clusters: NGC 2264, $\beta$ Pictoris, IC 2602, NGC $2451 \mathrm{~A}+\mathrm{B}, \alpha$ Persei, and Blanco 1 (see $§ 2.1 .2$ ). These data are shown in Figs. 18 and 19, along with models corresponding to the quoted age and metallicity of each cluster. The meaning of the figures in each row is the same as in Fig. 17. Each has been corrected for theoretical systematics by scaling relative to the calibrated Pleiades LDP described in $\S 4$, except NGC 2264, which has not yet suffered enough Li depletion for our systematic corrections to be meaningful.

The agreement between the data and models is good for each cluster. The dispersion present in the Pleiades is clearly a general phenomenon which develops between 6 and $20 \mathrm{Myr}$, and persists onto the ZAMS. This is consistent with an early origin of the Li spread, which our models predict develops between 8 and $15 \mathrm{Myr}$, and the subsequent termination of additional depletion until MS mechanisms kick in. There is a dip present at $4000 \mathrm{~K}$ in NGC 2264, which agrees well with the non-inflated models. At this same temperature, there are no data points that are consistent with the inflated upper envelope. This could reflect a time delay in the develop- 


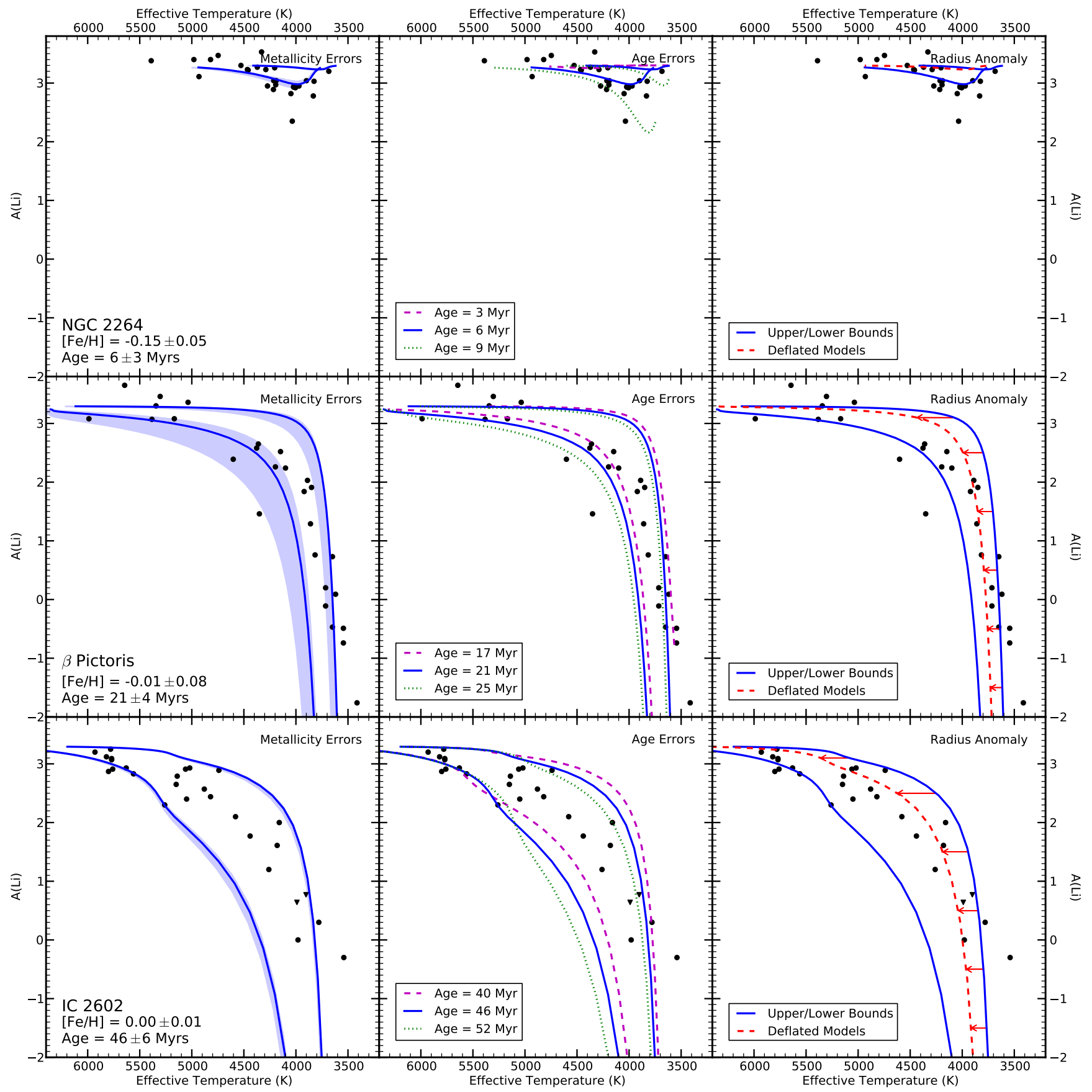

FIG. 18. - The same as in Fig. 17 for NGC 2264 (top), $\beta$ Pictoris (middle), and IC 2602 (bottom).

ment of the dispersion: at this young age, proto-stars are still contracting and spinning up, and therefore may not yet possess their ultimate radius anomaly. In the left columns, each cluster older than NGC 2264 falls within the bounds of our models for a $<1 \sigma[\mathrm{Fe} / \mathrm{H}]$ value. The Pleiades, $\beta$ Pictoris, NGC 2451, and Blanco 1 are all fit well by models generated with the fiducial $[\mathrm{Fe} / \mathrm{H}]$, but $\alpha$ Per is best fit by a metallicity close to solar. This agrees well with the $\alpha$ Per iron abundance measured by Boesgaard \& Friel (1990), who found an $[\mathrm{Fe} / \mathrm{H}]$ nearly identical to that of the near-solar Pleiades. The only clusters whose lower distribution is substantially different from the models is IC 2602, which appears to lie a few $100 \mathrm{~K}$ to the right of the predicted lower bound. While this could simply be due to the small number statistics of this data set, it could also be accounted for by assuming a slightly younger age for the cluster, as the bottom middle panel in Fig. 18 demonstrates. The agreement between these clusters and our predictions further support the arguments of $\S 6.1$, where we concluded that metallicity is a key factor in shaping cluster LDPs.

The central columns shows that the early-time LDP is quite sensitive to age. Li patterns rapidly evolve both in $\mathrm{A}(\mathrm{Li})$ and in $T_{\text {eff }}$ during their early stages, before stabilizing around $100 \mathrm{Myr}$. Each cluster appears consistent with its fiducial age, except IC 2602, whose LDP appears closer to $40 \mathrm{Myr}$ old than $46 \mathrm{Myr}$ old. This suggests that if the composition of a cluster is well known, and the 


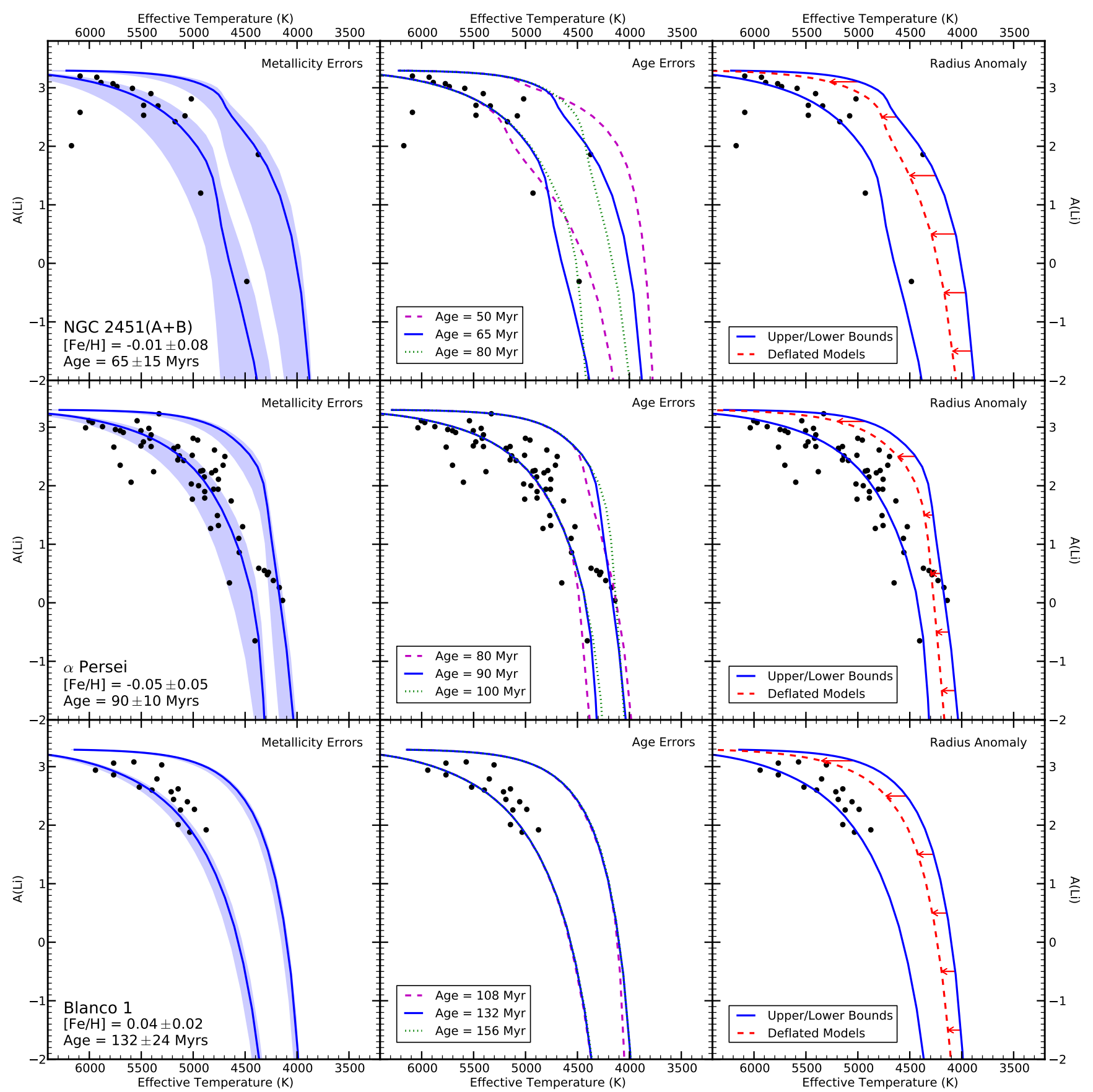

FIG. 19.- The same as in Fig. 17 for NGC 2451 A+B (top), $\alpha$ Persei (middle), and Blanco 1 (bottom).

distribution of pre-MS radii is taken into account, LDPs may be a viable method for inferring young cluster ages. This possibility has been doubted in the past, as the cause of the Li dispersion was not understood (i.e. Jeffries et al. 2009). However, these figures suggest that strong age constraints can be obtained through comparison with theory, even with our qualitative models. Furthermore, young cluster Li patterns are a useful way to measure the ages of clusters in the range 5-15 Myr, when our models predict that the dispersion develops. Bell et al. (2013) have recently proposed a revised age scale for several clusters younger than $20 \mathrm{Myr}$, which suggested new ages approximately double their previous literature values. $\mathrm{Li}$ observations could strongly discriminate be- tween their new ages and previous ages in several cases. For example, Bell et al. found an age of $12 \mathrm{Myr}$ for NGC 2362, up from 6.3 Myr (D'Antona \& Mazzitelli 1997). The former age implies significant depletion in some members, similar to the pattern of $\beta$ Pictoris, while the latter suggests only mild depletion and small dispersion, similar to the pattern of NGC 2264. This provides a strong test of the age scale of young clusters.

Finally, the right columns shows that even after the radius anomaly has vanished, the dispersion persists in all clusters older than $10 \mathrm{Myr}$. One might expect the red lines in the right column to bracket the upper envelope only in older clusters, and not in young clusters, whose members are still rapidly rotating. Indeed, the red 
line presents a superior qualitative representation of the upper envelope of NGC 2451(A+B), $\alpha$ Per and Blanco 1 , suggesting that, by $65 \mathrm{Myr}$, stars have spun down enough to suppress their radius anomalies. A single star in NGC 2451 lies substantially closer to the still-inflated upper envelope, as do several stars in $\alpha$ Per; this is not surprising, as lower mass stars spend more time on the pre-MS and spin down slower, and thus could still be rapidly rotating at this age. These clusters are likely in transition from an inflated to a deflated upper envelope. By contrast, the red line is clearly a worse fit to the upper envelopes of $\beta$ Pic and IC 2602 than the blue line, implying that the most inflated stars are still rotating quite rapidly at these young ages. The general picture that arises from this plot is that stars develop a Li dispersion around $10 \mathrm{Myr}$ due to a large range in radii at fixed mass, and converge on SSM predictions of radius and $T_{\text {eff }}$ sometime between 50 and $100 \mathrm{Myr}$.

It is worth re-emphasizing that these models were not calibrated to reproduce the Li patterns seen in Figs. 1619. The mixing-lengths used in our inflated models were chosen to qualitatively reproduce the range of radius anomalies seen in rapidly rotating systems. This implies that the Li patterns presented above are purely predictions of the model, not calibration points. The success of these models in reproducing the depletion patterns of many young and ZAMS clusters is very encouraging, and strongly implicates radius anomalies as a key ingredient in shaping young, cool star abundances. We note that the upper envelopes in Figs. 18 and 19 represent near-maximal inflation, and thus are almost certainly an optimistic prediction of the true upper envelope of the distribution. However, as described at the beginning of this section, several observational effects may artificially increase the measured $\mathrm{Li}$ abundance in these stars (e.g. line formation physics, rapid rotation, spots). If one were able to deconvolve the actual abundances from errors brought on by observational effects and Poisson noise, the maximum radius anomaly needed to reflect the upper envelope would likely decrease. Therefore, even in the event that $10-15 \%$ is an optimistic upper limit, this effect may produce a sufficient abundance spread.

It is also worth noting that several open clusters contain a population of $T_{\text {eff }} \sim 5500-6000 \mathrm{~K}$ stars that lie significantly below the mean trend. These can be seen in the Pleiades in Fig. 17, NGC $2451 \mathrm{~A}+\mathrm{B}$ and $\alpha$ Per in Fig. 19, the Hyades in Fig. 1, and in many other clusters (i.e. IC 4665 - Jeffries et al. 2009; NGC 3532 - S03). These stars cannot be explained through depletion mechanisms considered in this work, since the one non-standard effect we have included can only suppress Li depletion. Non-member contamination cannot be completely ruled out, even when objects are consistent with membership, which may explain a portion of these objects. However, if some are indeed cluster members, additional physical processes must have influenced their depletion history, the exploration of which is beyond the scope of this paper. We therefore call attention to these objects as possibly reflecting a distinct Li depletion pathway, but do not speculate about their nature. Further observational work, particularly a robust confirmation of cluster membership, should be undertaken to investigate these objects.

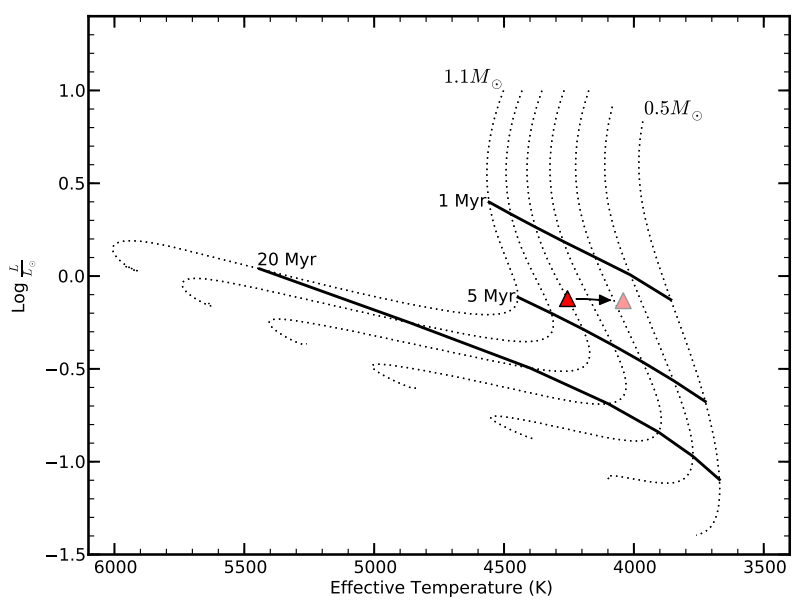

FIG. 20.- The impact of the radius anomaly for the location of pre-MS stars on the HR diagram. Black dotted lines represent the location of SSM evolutionary tracks for $\mathrm{M}=1.1-0.5 M_{\odot}$, and the black solid lines show SSM isochrones at 3 ages. The solid red triangle shows the HR diagram location of a $0.9 M_{\odot}, 3 \mathrm{Myr}$ old SSM, while the faded triangle shows the same model with a radius inflation of $\sim 10 \%$. Underestimates of $30 \%$ and $40 \%$ in the mass and age would result if the stellar parameters of the inflated star were inferred with these standard pre-MS models.

\subsubsection{Consequences of the Radius Anomaly in Young Clusters}

The presence of radius anomalies in young systems has consequences for some astrophysical measurements. Fig. 20 shows pre-MS tracks and isochrones for standard stellar models. The solid red triangle represents the HR diagram location of a $0.9 M_{\odot}, 3$ Myr old model with standard radius, and the faded red triangle represents the HR diagram location of a star with the same mass and age, but with a radius anomaly of $\sim 10 \%$. As can be seen, a puffed-up envelope reduces the $T_{\text {eff }}$ of a stellar model at fixed mass, while the luminosity remains nearly invariant. This effect shift pre-MS isochrones towards cooler temperatures. The Kelvin-Helmholtz time of inflated stars is also longer, since their initial gravitational potential energy is larger. This shifts the inflated isochrones to younger ages. The result is that while the faded triangle is $0.9 M_{\odot}$ and $3 \mathrm{Myr}$ old, isochrone matching would infer a mass of $\sim 0.65 M_{\odot}$ and an age of $\sim$ $1.7 \mathrm{Myr}$, resulting in errors of $\sim 30 \%$ and $40 \%$ respectively for a $10 \%$ radius anomaly. This is an important effect, since the fundamental parameters of young stars are routinely measured by placement on a grid of pre-MS isochrones. If this method is used to derive the masses and ages of individual stars, inflated objects will be systematically older and more massive than inferred.

This could have important consequences for several calculations. First, many studies use masses derived for preMS stars to measure the initial mass function (IMF) of the stellar distribution. These studies rely on the oneto-one mapping between absolute magnitudes and mass predicted by standard theory to derive stellar properties. However, the range of surface activity in young systems implies the presence of a range of radius anomalies. This breaks down the one-to-one mapping between observables and stellar parameters, and can severely bias determinations of the cluster mass function. The presence of inflated stars will tend to scatter individual mass measurements towards low values relative to the true mass, 
causing the inferred IMFs to be bottom-heavy. The impact of this effect can be minimized by studying older clusters, whose rotation distribution has converged to the rotation-mass-age relationship predicted by gyrochronology (e.g. Lachaume et al. 1999; Barnes 2007; Epstein \& Pinsonneault 2014). Other studies use the locus of data of pre-MS stars on the HR diagram to estimate the age of clusters. If a range of radius anomalies is present, the data will not fall on a single isochrone, but instead be spread out between a standard isochrone and a maximally inflated isochrone. If a single isochrone is fit to the mean data trend for low mass stars on the pre-MS, the age could be underestimated by up to $40 \%$. This error could be minimized by determining the SSM isochrone that best fits the hot side of the data (i.e. the slowest rotating stars), as radius anomalies scatter data towards cooler effective temperatures. Fortunately, this behavior does not impact measurements that use high-mass stars to determine ages.

Inflated radii may also bias cluster age measurements obtained through the LDB technique. To test this, we run standard and inflated stellar models of fully convective stars to the ZAMS. We find that if a star is inflated on the pre-MS, the onset of $\mathrm{Li}$ depletion will be delayed compared to a SSM star of equal mass, and thus systematically bias ages to younger values. In particular, if a range of radius anomalies are present in a young cluster, the transition from Li-rich to Li-poor will be fuzzed out over a characteristic mass range, since the transition mass at fixed age depends on the degree of inflation. Our findings are consistent with the studies of Burke et al. (2004), and MacDonald \& Mullan (2010). However, they are not consistent with Yee \& Jensen (2010), who argue that pre-MS inflation may lead to LDB estimates that are older than the true ages. They reach this conclusion by noting that inflated stars have a lower $T_{\text {eff }}$, which causes FCS masses to be systematically underestimated by SSM isochrones. This will in turn lower the inferred transition mass, leading to an older inferred age. However, their analysis assumed that the onset of Li destruction is not altered by inflation, which our models strongly rule out. Delaying Li burning drives age estimates to lower values, canceling out to some degree the impact of the effect they describe. This demonstrates a weakness in using $T_{\text {eff }}$ as a mass proxy: unless you know the radius anomaly of a given star, you cannot use its temperature to derive the mass. There is a similar weakness that arises when luminosity is used as a mass proxy: changes to the mass-radius relationship, and a possible increase of the spot filling-factor through stronger magnetic activity, can impact bolometric corrections. A full investigation of the impact of inflation on LDB ages must therefore take three important effects into account: the delay in the onset of Li destruction in inflated stars, the decreased $T_{\text {eff }}$ accompanying a larger radius, and changing bolometric corrections due to radius anomalies and magnetic activity. We suggest that future LDB studies aim to identify inflated stars by their CMD locations, and use both absolute and differential photometry to provide the best constraints on their masses. Finally, we note that we have used LDB ages for the clusters in this section. If these ages are incorrect, it could impact the agreement between open cluster data and our models. However, we find that a reduction of $\alpha$ of 0.8 produces a maximum age error of $\sim 10 \%$ which, given the minor sensitivity of LDPs to age (center columns, Figs. 1618), does not change the qualitative conclusions of this section.

\subsubsection{Summary and Future Prospects}

We have proposed a explanation for the cool star Li abundance spread present in young clusters. This dispersion would arise naturally in the presence of a radius dispersion at fixed mass during the pre-MS. Interferometric observations of single stars and precision tests of stellar models using detached eclipsing binaries evince the existence of inflated radii in some chromospherically active stars. Activity also correlates with rotation, so the most rapidly rotating stars likely possess the largest radius anomalies. If these stars are inflated on the preMS, the temperature at the base of their CZs will be less than their slowly rotating counterparts, strongly inhibiting the rate of pre-MS Li depletion, and causing them to be over-abundant in Li on the ZAMS.

We imposed a radius anomaly on our models of $\sim$ $5-15 \%$, consistent with the upper envelope of observed anomalies, by reducing the efficiency of convection. $\mathrm{Li}$ depletion is dramatically inhibited in these models, leading to a $>1$ dex spread at some temperatures. These models succeeded in qualitatively predicting the upper envelope of the $\mathrm{Li}$ distribution of the Pleiades. Furthermore, this theory naturally predicts the rotationabundance correlation seen in cool Pleiads. We then extended our models to 6 additional young clusters, and found good agreement between the empirical evolution of Li patterns from 0-120 Myr and our models. Since the inflated models were not calibrated to reproduce the $\mathrm{Li}$ patterns of these clusters, and instead were calibrated to match the observed upper envelope of radius anomalies in rapidly rotating systems, their success in reproducing the LDPs of several young clusters strongly supports the validity of this theory. Finally, we ended with a discussion of some effects that inflated radii could have on pre-MS isochrones, stellar IMF measurements, and ages determined through the LDB technique.

Improved measurements of $\mathrm{Li}$ and rotation in clusters shortly before and after the epoch of SSM burning will provide a crucial test of this theory. Equal mass stars with different rotation rates will show similar Li abundances before $5 \mathrm{Myr}$, and radically different abundances by $\sim 15 \mathrm{Myr}$, with the slower rotating stars showing larger degrees of depletion. The $13 \mathrm{Myr}$ old cluster $\mathrm{h}$ Per is a prime target for studying the late-time dispersion evolution, as a rich rotation data set has recently become available (Moraux et al. 2013). Fast and slow rotating stars of equal mass can be targeted, providing a measure of both the Li spread at fixed $T_{\text {eff }}$, and of the correlation between $\mathrm{Li}$ abundance and rotation at $13 \mathrm{Myr}$. Further theoretical work remains to solidify the predictions of this theory. In particular, future work should explicitly tie the radius anomaly to stellar rotation rates through empirical correlations (i.e. Stassun et al. 2012), and account for the simultaneous effects of inflation and rotational mixing. In Paper II, we will present calculation of non-inflated, rapidly rotating stellar models that greatly over-predict Li depletion on the early pre-MS. If true pre-MS stars are in fact inflated as described above, this extra mixing may be suppressed such that they enter 
the ZAMS with the correct abundances. Predictions of the rotating ZAMS Li pattern hinge on the interplay between the suppression of depletion by the inflated radius and the enhancement of depletion by meridional flows and shear instabilities. The relative strengths of these counteracting effects are difficult to anticipate without detailed modeling, but are crucial for establishing the validity of this theory.

\section{CONCLUSIONS}

We have attempted to reconcile SSMs with observations at the ZAMS, and use their predictions to uncover the behavior of Li on the MS. Through our investigation, we have reached several conclusions about SSM Li predictions, the progression of Li destruction on the MS, and the development of dispersion in cluster Li patterns.

- Pre-MS Li depletion in solar analogs is accurately predicted by standard stellar models.

SSM Li depletion on the pre-MS is highly sensitive to the assumed input physics. Adopting alternate choices of physical inputs, such as the cluster metal content, the equation of state tables, the opacity tables, and the ${ }^{7} \operatorname{Li}(p, \alpha) \alpha$ interaction cross-section, induces substantial changes in the predicted Li pattern at the ZAMS. We measured the impact of each physical input on pre-MS Li destruction, and derived an error band. Within this band, the distribution of solar analogs in the near-ZAMS Pleiades agrees with standard predictions. This allows us to perform an ad hoc calibration of the physics in our models, by forcing them to accurately predict the $\mathrm{Li}$ pattern of the Pleiades. With these calibrated models, we can predict the ZAMS distribution of other clusters by applying the metallicity scalings predicted by standard theory. This allows us to detrend the metallicitydependent pre-MS depletion out of empirical cluster data, leaving behind the depletion occurring on the MS, which is insensitive to metallicity and input physics.

- A radius dispersion on the pre-MS is the cause of the cool star Li dispersion in young clusters.

Some chromospherically active stars are known to possess inflated radii relative to standard predictions. We present calculations that demonstrate this radius inflation will decrease the temperature at the base of the $\mathrm{CZ}$, leading to a severe suppression of the rate of $\mathrm{Li}$ depletion during the pre-MS. The wide range of chromospheric activity at fixed mass in the cool Pleiads then naturally explains the spread in $\mathrm{Li}$ abundances. Correlations between chromospheric activity and rotation have also been reported, demonstrating that the most abundant stars are also the most rapidly rotating. We calculate inflated stellar models, and show that they accurately reproduce the width of the empirical Li spread in the Pleiades, as well as 6 additional young clusters. This strongly implies that a radius dispersion on the pre-MS is the underlying cause of the spread in lithium. We further explored some consequences of this effect for pre-MS isochrones and age measurements of young clusters. Finally, we suggest directions for future theoretical work, and propose dual $\mathrm{Li}$ and rotation data sets of $\sim 8-15$ Myr old clusters as an observational test.

- Dispersion is a general feature of Li patterns on the MS.
We detrend the strong pre-MS mass trends out of the full suite of clusters considered by SR05, and infer the dispersion within $T_{\text {eff }}$ bins centered at $6200 \mathrm{~K}, 5900 \mathrm{~K}$, $5600 \mathrm{~K}$, and $5300 \mathrm{~K}$. Within each bin, a dispersion is found to emerge in some clusters during the first $200 \mathrm{Myr}$. However, the evolution of this dispersion over time depends on the bin. The dispersion present at $100 \mathrm{Myr}$ shows little evolution for the $6200 \mathrm{~K}$ bin, but a marginal rising trend is seen in the $5900 \mathrm{~K}$ bin, a more convincing rising trend is seen in the $5600 \mathrm{~K}$ bin, and a decreasing trend is seen in the $5300 \mathrm{~K}$ bin. The degree of dispersion can differ between clusters of equal age. This complicated picture likely points to true cosmic variance in the initial conditions of open clusters. Finally, we show that the depletion mass trends identified by SR05 are stronger in anomaly space than in absolute space.

- The rate of MS Li depletion is a strong function of mass.

We isolated the MS depletion signal from two wellstudied MS clusters, the Hyades and M67, by subtracting the predicted ZAMS depletion factors from the empirical data of the clusters. Then, we measured the average rate of MS depletion over their lifetimes by comparing the median abundances of the clusters at several temperatures. This is the first measure of MS Li depletion that is not biased by $[\mathrm{Fe} / \mathrm{H}]$-dependent, differential pre-MS depletion. We find that low mass stars deplete Li significantly faster than high mass stars on the MS, placing strong constraints on propose mechanisms of MS mixing. We also find that the rate of MS depletion decays over time, in agreement with previous work. These are both robust conclusions, since they persist even in the presence of significant differences in the initial $\mathrm{Li}$ abundance of our benchmark clusters.

- Cluster metallicity is crucial in shaping ZAMS Li abundance patterns.

Some authors have argued that empirical studies of open clusters rule out a strong dependence of pre-MS Li depletion on metallicity. We show that an outstanding case for this argument, involving three 2 Gyr old clusters with dissimilar composition but similar Li patterns, does not rule out the theoretically predicted pre-MS metallicity dependence. We then analyze two $\sim 600 \mathrm{Myr}$ old clusters that differ in $[\mathrm{Fe} / \mathrm{H}]$ by 0.2 dex, and show substantial differences in their cool star Li patterns. Their absolute Li distributions are shown to be different at high confidence. When the metallicity dependence of SSMs is corrected for, they become statistically indistinguishable. Our models accurately predicted the difference in abundances of these clusters, strongly implicating metallicity as an important factor. Since cluster LDPs are sensitive to metallicity, sizable Li data sets can be used to estimate the metallicity of their host clusters by comparison with a similar aged cluster of well known composition. We apply a simple implement ion of this method to the Praesepe cluster, and find a best-fit $[\mathrm{Fe} / \mathrm{H}]=+0.16$, in $1 \sigma$ agreement with the recent high-resolution spectroscopic measurement of $+0.12 \pm 0.04$ (Boesgaard et al. 2013).

The authors thank Keivan Stassun for his helpful comments on the manuscript. 


\section{REFERENCES}

Alexander, D. R., \& Ferguson, J. W. 1994, ApJ, 437, 879

Allard, F., Hauschildt, P. H., Alexander, D. R., \& Starrfield, S. 1997, ARA\&A, 35, 137

An, D., Terndrup, D. M., Pinsonneault, M. H., et al. 2007, ApJ, 655,233

Anders, E., \& Grevesse, N. 1989, Geochim. Cosmochim. Acta, 53, 197

Andronov, N., \& Pinsonneault, M. H. 2004, ApJ, 614, 326

Anthony-Twarog, B. J., Deliyannis, C. P., Twarog, B. A., Croxall, K. V., \& Cummings, J. D. 2009, AJ, 138, 1171

Asplund, M., Grevesse, N., Sauval, A. J., \& Scott, P. 2009, ARA\&A, 47, 481

Balachandran, S. 1995, ApJ, 446, 203

Balachandran, S. C., Mallik, S. V., \& Lambert, D. L. 2011, MNRAS, 410, 2526

Baraffe, I., \& Chabrier, G. 2010, A\&A, 521, A44

Barnes, S. A. 2007, ApJ, 669, 1167

Basri, G., Marcy, G. W., \& Graham, J. R. 1996, ApJ, 458, 600

Bell, C. P. M., Naylor, T., Mayne, N. J., Jeffries, R. D., \& Littlefair, S. P. 2013, MNRAS, 434, 806

Berger, D. H., Gies, D. R., McAlister, H. A., et al. 2006, ApJ, 644 , 475

Bildsten, L., Brown, E. F., Matzner, C. D., \& Ushomirsky, G. 1997, ApJ, 482, 442

Binks, A. S., \& Jeffries, R. D. 2013, MNRAS, L192

Boesgaard, A. M., \& Steigman, G. 1985, ARA\&A, 23, 319

Boesgaard, A. M., \& Tripicco, M. J. 1986, ApJ, 302, L49

Boesgaard, A. M., \& Friel, E. D. 1990, ApJ, 351, 467

Boesgaard, A. M., Roper, B. W., \& Lum, M. G. 2013, ApJ, 775, 58

Bouvier, J. 1990, AJ, 99, 946

Bouvier, J., Rigaut, F., \& Nadeau, D. 1997, A\&A, 323, 139

Boyajian, T. S., McAlister, H. A., Baines, E. K., et al. 2008, ApJ, 683,424

Boyajian, T. S., von Braun, K., van Belle, G., et al. 2012, ApJ, 757,112

Burke, C. J., Pinsonneault, M. H., \& Sills, A. 2004, ApJ, 604, 272

Cargile, P. A., James, D. J., \& Jeffries, R. D. 2010, ApJ, 725, L111

Carlsson, M., Rutten, R. J., Bruls, J. H. M. J., \& Shchukina, N. G. 1994, A\&A, 288, 860

Casagrande, L., Flynn, C., Portinari, L., Girardi, L., \& Jimenez, R. 2007, MNRAS, 382, 1516

Casagrande, L., Ramírez, I., Meléndez, J., Bessell, M., \& Asplund M. 2010, A\&A, 512, A54

Castro, M., Do Nascimento, J. D., Jr., Biazzo, K., Meléndez, J., \& de Medeiros, J. R. 2011, A\&A, 526, A17

Chaboyer, B., Demarque, P., \& Pinsonneault, M. H. 1995, ApJ, 441,876

Chabrier, G., Gallardo, J., \& Baraffe, I. 2007, A\&A, 472, L17

Clausen, J. V., Bruntt, H., Claret, A., et al. 2009, A\&A, 502, 253

Cummings, J. 2011, Ph.D. Thesis,

Cummings, J. D., Deliyannis, C. P., Anthony-Twarog, B., Twarog, B., \& Maderak, R. M. 2012, AJ, 144, 137

Cyburt, R. H., Fields, B. D., \& Olive, K. A. 2004, Phys. Rev. D, 69,123519

Cyburt, R. H., Fields, B. D., \& Olive, K. A. 2008, JCAP, 11, 12

D'Antona, F., \& Mazzitelli, I. 1994, ApJS, 90, 467

D'Antona, F., \& Mazzitelli, I. 1997, Mem. Soc. Astron. Italiana, 68,807

D'Orazi, V., \& Randich, S. 2009, A\&A, 501, 553

Dahm, S. E. 2008, Handbook of Star Forming Regions, Volume I, 966

Demory, B.-O., Ségransan, D., Forveille, T., et al. 2009, A\&A, 505, 205

Dobbie, P. D., Lodieu, N., \& Sharp, R. G. 2010, MNRAS, 409, 1002

Duncan, D. K., \& Jones, B. F. 1983, ApJ, 271, 663

Eggenberger, P., Haemmerlé, L., Meynet, G., \& Maeder, A. 2012, A\&A, 539, A70

Epstein, C. R., \& Pinsonneault, M. H. 2014, ApJ, 780, 159

Feiden, G. A., \& Chaboyer, B. 2012, ApJ, 757, 42

Feiden, G. A., \& Chaboyer, B. 2013, ApJ, 779, 183

Ferguson, J. W., Alexander, D. R., Allard, F., et al. 2005, ApJ, 623,585
Fleming, T. A., Gioia, I. M., \& Maccacaro, T. 1989, ApJ, 340, 1011 Ford, A., Jeffries, R. D., \& Smalley, B. 2005, MNRAS, 364, 272

François, P., Pasquini, L., Biazzo, K., Bonifacio, P., \& Palsa, R. 2013, A\&A, 552, A136

Grevesse, N., \& Sauval, A. J. 1998, Space Sci. Rev., 85, 161

Hartman, J. D., Gaudi, B. S., Pinsonneault, M. H., et al. 2009, ApJ, 691, 342

Hartman, J. D., Bakos, G. Á., Kovács, G., \& Noyes, R. W. 2010 MNRAS, 408, 475

Heiter, U., Soubiran, C., Netopil, M., \& Paunzen, E. 2013 , arXiv: 1311.2306

Herbig, G. H. 1965, ApJ, 141, 588

Herbst, W., Bailer-Jones, C. A. L., \& Mundt, R. 2001, ApJ, 554, L197

Herbst, W., Bailer-Jones, C. A. L., Mundt, R., Meisenheimer, K., \& Wackermann, R. 2002, A\&A, 396, 513

Hünsch, M., Weidner, C., \& Schmitt, J. H. M. M. 2003, A\&A, 402, 571

Hünsch, M., Randich, S., Hempel, M., Weidner, C., \& Schmitt, J. H. M. M. 2004, A\&A, 418, 539

Iben, I., Jr. 1965, ApJ, 141, 993

Iglesias, C. A., \& Rogers, F. J. 1996, ApJ, 464, 943

Irwin, J., \& Bouvier, J. 2009, IAU Symposium, 258, 363

Irwin, J., Berta, Z. K., Burke, C. J., et al. 2011, ApJ, 727, 56

Jeffries, R. D. 1999, MNRAS, 304, 821

Jeffries, R. D., \& James, D. J. 1999, ApJ, 511, 218

Jeffries, R. D. 2000, Stellar Clusters and Associations: Convection, Rotation, and Dynamos, 198, 245

Jeffries, R. D., Totten, E. J., Harmer, S., \& Deliyannis, C. P. 2002, MNRAS, 336, 1109

Jeffries, R. D., Jackson, R. J., James, D. J., \& Cargile, P. A. 2009, MNRAS, 400, 317

Johnson, H. L., \& Knuckles, C. F. 1955, ApJ, 122, 209

Jones, B. F., Fischer, D., \& Soderblom, D. R. 1999, AJ, 117, 330

Koenigl, A. 1991, ApJ, 370, L39

King, J. R., Krishnamurthi, A., \& Pinsonneault, M. H. 2000, AJ, 119,859

King, J. R., Schuler, S. C., Hobbs, L. M., \& Pinsonneault, M. H. 2010, ApJ, 710, 1610

Kraft, R. P. 1967, ApJ, 150, 551

Kraus, A. L., Tucker, R. A., Thompson, M. I., Craine, E. R., \& Hillenbrand, L. A. 2011, ApJ, 728, 48

Kurucz, R. L. 1979, ApJS, 40, 1

López-Morales, M., \& Ribas, I. 2005, ApJ, 631, 1120

López-Morales, M. 2007, ApJ, 660, 732

Lachaume, R., Dominik, C., Lanz, T., \& Habing, H. J. 1999, A\&A, 348,897

Lamia, L., Spitaleri, C., La Cognata, M., Palmerini, S., \& Pizzone, R. G. 2012, A\&A, 541, A158

Lyngå, G. 1985, IAUS, 106, 143

Macdonald, J., \& Mullan, D. J. 2010, ApJ, 723, 1599

MacDonald, J., \& Mullan, D. J. 2012, MNRAS, 421, 3084

Margheim, S. J., Deliyannis, C. P., King, J. R., \& Steinhauer, A. 2002, Bulletin of the American Astronomical Society, 34, \#124.03

Margheim, S. J. 2007, Ph.D. Thesis,

Meibom, S., Mathieu, R. D., Stassun, K. G., Liebesny, P., \& Saar, S. H. 2011, ApJ, 733, 115

Mendoza, C., Seaton, M. J., Buerger, P., et al. 2007, MNRAS, 378, 1031

Montalbán, J., \& Schatzman, E. 2000, A\&A, 354, 943

Montgomery, K. A., Marschall, L. A., \& Janes, K. A. 1993, AJ, 106,181

Morales, J. C., Ribas, I., \& Jordi, C. 2008, A\&A, 478, 507

Moraux, E., Artemenko, S., Bouvier, J., et al. 2013, A\&A, 560, A13

Mullan, D. J., \& MacDonald, J. 2001, ApJ, 559, 353

Pace, G., Pasquini, L., \& François, P. 2008, A\&A, 489, 403

Pace, G., Castro, M., Meléndez, J., Théado, S., \& do Nascimento, J.-D., Jr. 2012, A\&A, 541, A150

Palla, F., \& Stahler, S. W. 1992, ApJ, 392, 667

Pasquini, L., Biazzo, K., Bonifacio, P., Randich, S., \& Bedin, L. R. 2008, A\&A, 489, 677 
Perryman, M. A. C., Brown, A. G. A., Lebreton, Y., et al. 1998, A\&A, 331, 81

Pinsonneault, M. H., Kawaler, S. D., Sofia, S., \& Demarque, P. 1989, ApJ, 338, 424

Pinsonneault, M. H., Kawaler, S. D., \& Demarque, P. 1990, ApJS, 74,501

Pinsonneault, M. 1997, ARA\&A, 35, 557

Popper, D. M. 1997, AJ, 114, 1195

Preibisch, T., Kim, Y.-C., Favata, F., et al. 2005, ApJS, 160, 401

Press, W. H. 1981, ApJ, 245, 286

Prisinzano, L., \& Randich, S. 2007, A\&A, 475, 539

Randich, S., Pasquini, L., \& Pallavicini, R. 2000, A\&A, 356, L25

Randich, S., Pallavicini, R., Meola, G., Stauffer, J. R., \& Balachandran, S. C. 2001, A\&A, 372, 862

Randich, S., Pace, G., Pastori, L., \& Bragaglia, A. 2009, A\&A, 496, 441

Rebull, L. M., Stauffer, J. R., Megeath, S. T., Hora, J. L., \& Hartmann, L. 2006, ApJ, 646, 297

Ribas, I. 2003, A\&A, 398, 239

Richer, J., \& Michaud, G. 1993, ApJ, 416, 312

Rogers, F. J., \& Nayfonov, A. 2002, ApJ, 576, 1064

Rogers, F. J., Swenson, F. J., \& Iglesias, C. A. 1996, ApJ, 456, 902

Ryan, S. G. 2000, MNRAS, 316, L35

Sacco, G. G., Randich, S., Franciosini, E., Pallavicini, R., \& Palla, F. 2007, A\&A, 462, L23

Salaris, M., Weiss, A., \& Percival, S. M. 2004, A\&A, 414, 163

Saumon, D., Chabrier, G., \& van Horn, H. M. 1995, ApJS, 99, 713

Schuler, S. C., King, J. R., Fischer, D. A., Soderblom, D. R., \& Jones, B. F. 2003, AJ, 125, 2085

Sestito, P., Randich, S., Mermilliod, J.-C., \& Pallavicini, R. 2003, A\&A, 407, 289

Sestito, P., Randich, S., \& Pallavicini, R. 2004, A\&A, 426, 809

Sestito, P., \& Randich, S. 2005, A\&A, 442, 615

Shen, Z.-X., Jones, B., Lin, D. N. C., Liu, X.-W., \& Li, S.-L. 2005 , ApJ, 635, 608

Skumanich, A. 1972, ApJ, 171, 565

Soderblom, D. R., Jones, B. F., Balachandran, S., et al. 1993, AJ, 106, 1059

Soderblom, D. R., Fedele, S. B., Jones, B. F., Stauffer, J. R., \& Prosser, C. F. 1993, AJ, 106, 1080

Soderblom, D. R., King, J. R., Siess, L., Jones, B. F., \& Fischer, D. 1999, AJ, 118, 1301

Soderblom, D. R., Laskar, T., Valenti, J. A., Stauffer, J. R., \& Rebull, L. M. 2009, AJ, 138, 1292
Spite, F., \& Spite, M. 1982, A\&A, 115, 357

Stassun, K. G., Mathieu, R. D., Mazeh, T., \& Vrba, F. J. 1999, AJ, 117, 2941

Stassun, K. G., van den Berg, M., Feigelson, E., \& Flaccomio, E. 2006, ApJ, 649, 914

Stassun, K. G., Mathieu, R. D., \& Valenti, J. A. 2007, ApJ, 664, 1154

Stassun, K. G., Kratter, K. M., Scholz, A., \& Dupuy, T. J. 2012, ApJ, 756, 47

Stauffer, J. R., Hartmann, L., Soderblom, D. R., \& Burnham, N. 1984, ApJ, 280, 202

Stauffer, J. R., Schultz, G., \& Kirkpatrick, J. D. 1998, ApJ, 499, L199

Stauffer, J. R., Barrado y Navascués, D., Bouvier, J., et al. 1999, ApJ, 527, 219

Steinhauer, A. 2003, Ph.D. Thesis,

Strobel, A. 1991, Astronomische Nachrichten, 312, 177

Stuik, R., Bruls, J. H. M. J., \& Rutten, R. J. 1997, A\&A, 322, 911

Swenson, F. J., \& Faulkner, J. 1992, ApJ, 395, 654

Takeda, Y., Honda, S., Ohnishi, T., et al. 2013, PASJ, 65, 53

Taylor, B. J. 2007, AJ, 133, 370

Terndrup, D. M., Pinsonneault, M., Jeffries, R. D., et al. 2002, ApJ, 576, 950

Terrien, R. C., Fleming, S. W., Mahadevan, S., et al. 2012, ApJ, 760, L9

Thorburn, J. A., Hobbs, L. M., Deliyannis, C. P., \& Pinsonneault, M. H. 1993, ApJ, 415, 150

Tognelli, E., Degl'Innocenti, S., \& Prada Moroni, P. G. 2012, A\&A, 548, A41

Torres, G., \& Ribas, I. 2002, ApJ, 567, 1140

Torres, C. A. O., Quast, G. R., da Silva, L., et al. 2006, A\&A, 460, 695

Torres, G., Andersen, J., \& Giménez, A. 2010, A\&A Rev., 18, 67

Viana Almeida, P., Santos, N. C., Melo, C., et al. 2009, A\&A, 501, 965

Villanova, S., Carraro, G., \& Saviane, I. 2009, A\&A, 504, 845

Wilson, O. C. 1966, ApJ, 144, 695

Yee, J. C., \& Jensen, E. L. N. 2010, ApJ, 711, 303

Zahn, J.-P. 1992, A\&A, 265, 115

Zappala, R. R. 1972, ApJ, 172, 57 